BUHEP-93-5, USC-93/003, LPM-93-07

\title{
Massless Flows I: the sine-Gordon and $O(n)$ models
}

\author{
P. Fendley\#, H. Saleur ${ }^{\dagger}$ and Al.B. Zamolodchikov* \\ \# Department of Physics, Boston University \\ 590 Commonwealth Avenue, Boston, MA 02215, USA \\ ${ }^{\dagger}$ Department of Physics, University of Southern California \\ Los Angeles, CA 90089, USA \\ *Laboratoire de Physique Mathématique \\ Université Montpellier 2, Montpellier, France
}

The massless flow between successive minimal models of conformal field theory is related to a flow within the sine-Gordon model when the coefficient of the cosine potential is imaginary. This flow is studied, partly numerically, from three different points of view. First we work out the expansion close to the Kosterlitz-Thouless point, and obtain roaming behavior, with the central charge going up and down in between the UV and IR values of $c=1$. Next we analytically continue the Casimir energy of the massive flow (i.e. with real cosine term). Finally we consider the lattice regularization provided by the $O(n)$ model in which massive and massless flows correspond to high- and low-temperature phases.

A detailed discussion of the case $n=0$ is then given using the underlying $N=2$ supersymmetry, which is spontaneously broken in the low-temperature phase. The "index" $\operatorname{tr} F(-1)^{F}$ follows from the Painlevé III differential equation, and is shown to have simple poles in this phase. These poles are interpreted as occuring from level crossing (onedimensional phase transitions for polymers). As an application, new exact results for the connectivity constants of polymer graphs on cylinders are obtained. These results and points of view are used in the following paper to discuss the appropriate exact $S$-matrices and the resulting Casimir energies.

April 1993 


\section{Introduction}

Prompted by conformal field theory, there has been enormous progress in the last several years in understanding two-dimensional field theories off the critical point. For both massive and massless models, much has been understood by using perturbed conformal field theory. In the numerous cases where the model is integrable, a number of nonperturbative techniques have been extensively developed and applied. There are many massive models whose properties are very well understood, but there exist some very basic massless ones whose properties have not been fully explored.

A particular example is the flow between the $(t+1)^{\text {th }}$ and $t^{\text {th }}$ unitary models [1], which has been studied perturbatively for large $t$ [2] and for finite $t$ using the conjectured thermodynamic Bethe ansatz relations [3]. Moreover, this problem is related to an even less-understood model, which is the sine-Gordon model with an imaginary potential. This model is interesting in its own right, because at a particular coupling it describes the "dense" phase of self-avoiding polymers. These models are related because here and in many other cases, one can consider perturbed conformal field theories as restrictions of affine Toda theories, thus extending free-field representations of conformally invariant field theories. In the conformal case and the massive case, these restrictions are well known. The (Virasoro) minimal conformal field theories can obtained from a free boson [4] and the massive integrable perturbation of these theories by the $\Phi_{13}$ operator can be obtained from sine-Gordon [5,6]. In statistical-mechanical language, this is often called mapping the system onto a Coulomb gas [7]. The restriction is most easily understood in field theory using quantum-group structures [8-15], and to some extent parallels lattice-model constructions [16,17]. A very similar restriction applies also to the massive $O(n)$ model [18,19].

We discuss a similar truncation for massless flows, where both the UV and IR limits are conformally invariant. In this case, since both UV and IR fixed points are obtained by restriction of a free boson, one expects that the full trajectory is the restriction of an intriguing flow in sine-Gordon, with central charge equal to 1 at both extremities. This flow actually was first mentioned in [7], and related explicit field-theory calculations were done in [20]. We discussed it briefly in [21] where we presented numerical data with a "roaming" trajectory that violates the $c_{\text {eff }}$ conjecture. We describe in this paper and in the following our attempts to understand quantitatively this problem.

The models describing the flows between the minimal models are unitary, but the sine-Gordon model with imaginary potential has a Lagrangian that is not real, and hence 
is non-unitary. The non-unitarity allows a variety of interesting properties to appear, including level crossing, an increasing $c$-function, spontaneous breaking of supersymmetry, and singularities. While these features are part of what makes this model interesting, they also often make it difficult. In this paper and its sequel we will use a variety of methods to explore the situation. We believe that we have completed the picture for the minimal models; we will give the exact $S$-matrices and ground-state energy. However, we will see that there are still a number of unanswered questions in the case of sine-Gordon. The first paper contains detailed studies (including numerical) of the problem, excepting attempts to build a scattering theory. The second paper is then fully devoted to that question.

In section 2 we discuss the flow within sine-Gordon in an expansion close to the Kosterlitz-Thouless point. We recover the roaming trajectory with a pair of extrema of order $1 / t^{3}$, and discuss the behavior of the stress-energy tensor and alternate definitions of the central charge.

In section 3 we discuss in detail the lattice regularization of the problem provided by the $O(n)$ model on the honeycomb lattice flowing to the low temperature phase. A quantum-group symmetry present all along the flow is exhibited that will provide useful information about level crossings. A lattice derivation of the flow of independent sectors of the partition functions is also provided. This section may be skipped by the reader who is not interested in lattice questions.

In section 4 we discuss numerically the massless flow using two approaches. We discuss first the analytic continuation of the massive flow [21]. The first extremum of the central charge agrees nicely with the expansion around the Kosterlitz-Thouless point, but breaks down afterward. We also consider lattice data, which show the existence of multiple extrema for small enough $t$, a feature not seen in the large- $t$ expansion.

Section 5 contains a short discussion of the boundary case $n=-2$, which is exactly solvable by other methods. The behavior is somewhat pathological, but the appearance of singularities provides compelling evidence for similar behavior to occur at least at nearby values of $n$.

Section 6 contains a detailed discussion of the case $n=0$. This case is physically very important because it describes polymers, and is also quite amenable by analytic techniques due to the underlying $N=2$ supersymmetry. We find that $N=2$ supersymmetry is spontaneously broken; the theorem that ordinarily does not allow this does not apply as a result of non-unitarity (despite the non-unitarity, the described model is meaningful for condensed-matter physics). It is shown that an infinite number of level crossings separate 
the UV and IR fixed points. These crossings are studied analytically by calculating the index $\operatorname{tr} F(-1)^{F}$ 22, 23, where they show up as poles. The locations of these poles are found by solving the Painlevé III differential equation in the complex plane.

\section{Thermal flow: generalities and the large- $t$ expansion}

Consider the $(t-1)^{\text {th }}$ minimal unitary conformal field theory with central charge [24]

$$
c=1-\frac{6}{t(t+1)}
$$

where $t$ is an integer. As is well known, it can be obtained by twisting and reducing a Gaussian model [4] (a free scalar field $\phi$ ) which has coupling in "lattice-like" conventions $g=1+\frac{1}{t}[\overline{[}]$.

We perturb this critical theory by the thermal operator, which is identified in the conformal language as $\Phi_{13}$, and has left and right dimensions

$$
h=\bar{h}=\frac{t-1}{t+1} .
$$

Perturbation by $\Phi_{13}$ corresponds in the Gaussian model to a perturbation by the operator $\cos 2 \phi$ with weight, with respect to the untwisted stress energy tensor

$$
h=\bar{h}=\frac{t}{t+1} .
$$

The coupling constants are different due to the difference in conformal weights in the twisted and untwisted theory. If for the minimal model

$$
S_{\min }=S_{\min }^{*}+(\delta \beta) \int \Phi_{13} d^{2} x
$$

then

$$
S_{S G}=\int d^{2} x\left[\frac{g}{4 \pi}(\partial \phi)^{2}+(\delta \beta)^{1 / 2} \cos 2 \phi\right]
$$

The mass in the minimal model is proportional to $|\delta \beta|^{\frac{t+1}{4}}$, where $\delta \beta=\beta_{c}-\beta$ refers to distance from the critical inverse "temperature".

For $\delta \beta>0$ the minimal model is expected to flow to a trivial massive phase. From the Gaussian point of view, we indeed have the standard sine-Gordon model. It is integrable, with a known $S$-matrix, and only the massive soliton and antisoliton are in the spectrum for $t \geq 2$ [25]. From this $S$-matrix and the thermodynamic Bethe ansatz (TBA), relations 
can be written for the ground-state energy which restrict to the ones conjectured for the minimal models [3] when appropriately twisted [21].

When $\delta \beta<0$ the minimal model flows [1,2] to another conformal field theory with central charge in the infrared

$$
c=1-\frac{6}{t(t-1)}
$$

The corresponding situation in the sine-Gordon theory is a little unusual. It leads in (2.5) to a purely imaginary coupling, a non-unitary case that has already attracted some attention [7,20,26]. The behavior can be understood qualitatively as follows. Call $\lambda_{e}$ the coupling of the vertex operator $e^{i e \phi}$ in a general action with the Gaussian term plus perturbations. In (2.5) for instance we have $\lambda_{2}=\lambda_{-2}=\frac{1}{2}(\delta \beta)^{1 / 2}$. Approximate renormalization group equations can be written for the $\lambda_{e}$ and the coupling constant $g$ [27,28]. If $b$ is the rescaling parameter, one finds at first order

$$
\frac{d}{d b} \lambda_{e}=\left(2-\frac{e^{2}}{2 g}\right) \lambda_{e}
$$

and for the coupling constant

$$
\frac{1}{g} \frac{d}{d b} g=8 \pi^{2} \lambda_{e} \lambda_{-e} .
$$

No magnetic charges arise in these equations. Observe that $\cos 2 \phi$ is relevant for $g>1$, irrelevant otherwise.

In the case $\delta \beta>0, \lambda_{2}=\lambda_{-2}$ are real numbers. Then if initially $g>1$ as in the case here, $\lambda_{2}$ and $\lambda_{-2}$ both increase along the renormalization group flow (2.7). This increase contributes to increasing $g$ as well due to (2.8) and in turn to making $\cos 2 \phi$ even more relevant. So at this order, more and more charges \pm 2 are generated at large distance, and one expects (slightly changing the language) the "Coulomb gas" [7,27] to flow to a massive, plasma phase.

Now suppose $\delta \beta<0$. Initially, the magnitudes of both $\lambda_{2}$ and $\lambda_{-2}$ both increase in the renormalization. However, since they are purely imaginary, their product is negative, so due to (2.8) the coupling decreases. This decrease means that the operator $\cos 2 \phi$ becomes less relevant. One therefore expects that floating charges \pm 2 are present in large numbers at intermediate distances, but ultimately disappear in the IR. If this is so, the sine-Gordon model with imaginary coupling should flow back to a Gaussian model, although with a smaller coupling constant. This phenomena was first discussed in [7] in relation to the dense $O(n)$ model to be discussed in subsequent sections. 
There is a simple way of understanding this heuristically [5, 6, 20]. This is to postulate that the action for the conformal minimal model in (2.4) is in fact the Liouville action with a curvature term to represent the charge at infinity:

$$
S_{\min }^{*}=\int \frac{g}{4 \pi}\left[(\partial \phi)^{2}+i Q R \phi\right]+\lambda_{2} e^{2 i \phi}
$$

where $R$ is the scalar curvature. This model is reasonably well-understood (at least with $Q$ imaginary, in our conventions) and has a fixed point which is a conformal theory of central charge $c=1-6 g Q^{2}$. Thus the conjecture is that with appropriate choices of $g$ and $Q$ (real here), this action describes a minimal model. The operator with dimensions of $\Phi_{1,3}$ turns out to be $\exp (-2 i \phi)$. Thus to perturb the model, we add $\lambda_{-2} \int \exp (-2 i \phi)$ to the action, and obtain the sine-Gordon model (2.5) plus a curvature term. Thus in this sense, the actions (2.5) and (2.4) differ only by the curvature term. Calculations in field theory including the curvature term have been done for large $t$ in [20]; these indeed reproduce the flow of the central charge between successive minimal models. The sign of the perturbation is crucial: we can always redefine the coefficients of the exponential terms by shifting the field $\phi$, but the product $\lambda_{2} \lambda_{-2}$ does not change. Thus if this product is positive we are in the massive phase, while if it is negative we are in the massless phase.

\subsection{Large-t expansion}

It is possible to study this a little more quantitatively in the limit of large $t$. Setting $e=2$ in (2.7) and (2.8), both the fugacities and the renormalization group eigenvalue

$y_{e}=2-\frac{e^{2}}{2 g}$ are small in this limit, and we can simultaneously expand in both variables. At dominant order these two equations allow us to compute

$$
\frac{d}{d g} \frac{d g}{d b}=4(g-1)
$$

which we can integrate to

$$
\frac{d g}{d b}=2\left[(g-1)^{2}-\frac{1}{t^{2}}\right]
$$

where the integration constant is determined by the UV value $g_{U V}=1+\frac{1}{t}$. This beta function vanishes at another value of $g$, indeed smaller than one as expected, $g_{I R}=1-\frac{1}{t}$ (this value is in fact exact, but all we get here is a check of it at first order). The flow can be integrated to give

$$
g=\frac{g_{U V}+g_{I R} e^{4\left(b-b_{0}\right) / t}}{1+e^{4\left(b-b_{0}\right) / t}}
$$


and

$$
\lambda_{2}=-\lambda_{-2}=\frac{i}{\pi t} \frac{e^{2\left(b-b_{0}\right) / t}}{1+e^{4\left(b-b_{0}\right) / t}} .
$$

Both the running coupling constant $g$ and the fugacities exhibit the behavior qualitatively described in [7]. In particular there is a value of $b=b_{0}$ where the fugacities are extremal, so that their associated beta functions vanish. However, this is not an intermediate fixed point at this order, because the beta function for the coupling constant $g$ is not zero but instead extremal while $g$ passes through $g=1$.

Further information about this flow in the Gaussian model is obtained by considering the central charge. Recall that at first order the trace of the stress-energy tensor for the Gaussian model perturbed by vertex operators of charge $\pm e$ reads 28]

$$
\Theta=-2 \pi y_{e}\left(\lambda_{e} e^{i e \phi}+\lambda_{-e} e^{-i e \phi}\right)
$$

From this and the relation between the derivative of the running central charge defined in [1] and $\Theta$ we find the running central charge at distance $R$

$$
c(R)=1-6 \pi^{2} y_{e}\left(R^{y_{e}} \lambda_{e} R^{y_{e}} \lambda_{-e}\right),
$$

Put now $e=2$. When $\delta \beta>0$ the product of fugacities is positive, so $c$ decreases in agreement with the $c$-theorem. On the other hand, when $\delta \beta<0$ the $c$-theorem is not expected to hold due to the non-unitarity of the theory. 1 In fact, one has the opposite behavior; since the couplings are purely imaginary, $c$ initially increases above its UV value 1, going against a " $c$ eff-theorem" (this was observed in [26]). Ultimately, $c$ must return to 1 in the IR. At the order to which we are working, $c$ is given by

$$
c=1+\frac{12}{t^{3}} \frac{e^{4\left(b-b_{0}\right) / t}\left(1-e^{4\left(b-b_{0}\right) / t}\right)}{\left(1+e^{4\left(b-b_{0}\right) / t}\right)^{3}},
$$

and it exhibits a pair of extrema at $e^{4\left(b-b_{0}\right) / t}=(2 \pm \sqrt{3})$ with values

$$
c_{ \pm}=1 \pm \frac{2}{\sqrt{3}} \frac{1}{t^{3}}
$$

The $R$ dependence disappears at this order.

1 Here this non-unitarity manifests itself rather trivially by the negative sign of the two point function of $\Theta$. 
As a result of the vanishing of the beta functions for the fugacities, the two-point functions $\langle\Theta \Theta\rangle$ and $\langle T \Theta\rangle$ vanish when $g$ passes through $g=1$ at this order. It is likely that higher-order contributions give them a finite but small value. Indeed, assuming that the coupling $g$ evolves monotonically, its beta function never vanishes, and there cannot be any intermediate fixed point.

Since $c(R)$ is equal to one both in the UV and IR where the sine-Gordon model flows to a Gaussian model, for any value of $R$ it must exhibit at least a pair of extrema. Using the Callan-Symanzik equation

$$
R \frac{\partial c}{\partial R}+\beta_{g_{i}} \frac{\partial c}{\partial g_{i}}=0
$$

for coupling constants $g_{i}$ (here $g, \lambda$ ) we see, without any reference to a given order of perturbation, that at these extrema the derivative $\frac{\partial c}{\partial R}$ has to vanish, which in turn means vanishing of $\langle\Theta(0) \Theta(R)\rangle$. However we can expect a priori that the peculiar values of the couplings $g_{i}$ at the extrema depends on $R$ so there is no special intermediate point in the flow where $\langle\Theta(0) \Theta(R)\rangle$ would vanish for every $R$. It is possible for such a two-point function to vanish for some value of $R$ because of the non-unitarity of the theory.

Beyond this order, the perturbative study of the flow looks quite complicated. However, according to the arguments of [3], this model is integrable, and non-perturbative methods should be applicable. Recall indeed that in the massive flow, the running central charge $c(m R)$ ( $m$ is the soliton mass) for the sine-Gordon model was successfully calculated by using the TBA [21]. (As we will explain in section 4, the running central charge is defined via the Casimir effect on a cylinder.) It can also be computed in the large- $t$ expansion [2] and one finds at first order the same formula as (2.15) with $R=1$. The study of this function $c(m R)$ for finite $t$, and the search for its corresponding TBA, are the subjects of this paper and the next. An immediate result that can be obtained for finite $t$ is the exactness of $g_{I R}=1-\frac{1}{t}$. This follows most easily from the $O(n)$ model discretization, and symmetries (see the discussion of eq. 3.20). We therefore see that the massless flow in sine-Gordon should be observed for $t \geq 1$ only. In the standard parametrization this corresponds to $4 \pi \leq \beta_{S G}^{2} \leq 8 \pi$. In particular we do not expect this massless flow to have a semi-classical version since the latter is obtained as $\beta_{S G} \rightarrow 0$.

In the large- $t$ expansion we considered only $\lambda_{ \pm 2}$, but the renormalization group transformations generate higher charges as well. In the region where $\lambda_{ \pm 2}$ are found decreasing, they disappear for two reasons. On the one hand some pairs of opposite charges annihilate and contribute simply to renormalizing the coupling constant $g$. On the other hand pairs 
of identical charges coalesce to increase first of all $\lambda_{ \pm 4}$. Of course these are irrelevant with respect to the UV fixed point. Their equation of evolution is made of a damping term $y_{4} \lambda_{ \pm 4}$ and a source term proportional to $\lambda_{ \pm 2}^{2}$. So we expect $\lambda_{ \pm 4}$ to increase driven by the amount of \pm 2 charges, and subsequently to decrease when charges \pm 2 have disappeared. The picture generalizes to all higher charges. Therefore, provided the domains of evolution of these various charges are separated enough, one can expect more structure in $c(m R)$ than just a pair of extrema, but it is difficult to make quantitative predictions.

\section{3. $O(n)$ vertex model: Symmetries of the thermal flow}

We discuss in this section various properties of the $O(n)$ model on the honeycomb lattice. This model provides a convenient regularization of our problem when it flows to the low-temperature "dense" phase. It is also useful in identifying symmetries of the flow and the occurence of level crossings.

\section{1. $O(n)$ vertex model and its symmetries}

It is well known that a convenient lattice regularization for the sine-Gordon model and its restrictions is provided by the $O(n)$ model on the honeycomb lattice (the links of a hexagonal lattice). The original partition function in the absence of a magnetic field is

$$
\mathcal{Z}=\sum \beta^{M} n^{L}
$$

where the summation is taken over all configurations of self-avoiding, mutually avoiding loops that can be drawn on the lattice, $M$ is the number of lattice edges occupied by a piece of loop (monomer) and $L$ is the number of loops. As it stands, (3.1) is not defined locally. A local description is obtained as follows [7]. We still associate a weight $\beta$ to each monomer, but we do not give weights $n$ to loops any more. Instead we put an orientation on each loop, together with a weight $e^{ \pm i \pi e_{0} / 6}$ per left (right) turn. On a planar honeycomb lattice, the number of left minus the number of right turns of a closed loop is equal to \pm 6 . Therefore setting

$$
n=2 \cos \pi e_{0}
$$

we see that summing over all the possible orientations reproduces the desired weight $n$ per loop, now as a sum of local contributions. We refer to this model of oriented loops as the $O(n)$ vertex model, or vertex model. It has been given other names and is well known 
to be related in some limits to the Zamolodchikov-Fateev model and the Izergin-Korepin model; see [29]. As we will discuss below, the correspondence between (3.1) and the vertex model is a little more intricate in non-trivial topologies like the cylinder or torus.

The difference between the non-local and local formulations is manifest if one tries to write a transfer matrix. In the first case, "past" information must be taken into account: we need to know if two monomers on a given column were connected at former times (see [30] and references therein). In the second case, we simply have a vertex model with an $\check{R}$-matrix acting in the product of two spin-one representations, and the transfer matrix is the appropriate tensor product of these $\check{R}$ matrices. To see this consider propagation along one of the three equivalent directions in the honeycomb lattice. Following [31] we decompose the interactions into elementary three-leg vertices with weights

$$
\begin{aligned}
& \omega_{1}, \ldots, \omega_{7}=\beta^{-1}, \epsilon, \epsilon, \epsilon, \epsilon^{-1}, \epsilon^{-1}, \epsilon^{-1} \\
& \omega_{1}^{\prime}, \ldots, \omega_{7}^{\prime}=\beta^{-1}, \epsilon^{-1}, \epsilon^{-1}, \epsilon^{-1}, \epsilon, \epsilon, \epsilon
\end{aligned}
$$

where we set $\epsilon=e^{i \pi e_{0} / 6}$. As usual we can modify these weights (by a so-called gauge transformation) and leave the partition function invariant. The general transformation of interest reads

$$
\begin{aligned}
& \omega_{1}, \ldots, \omega_{7}=\beta^{-1}, \frac{c^{\prime} \epsilon}{a}, \frac{a^{\prime} \epsilon}{b}, \frac{b^{\prime} \epsilon}{c}, \frac{a^{\prime}}{c \epsilon}, \frac{b^{\prime}}{a \epsilon}, \frac{c^{\prime}}{b \epsilon} \\
& \omega_{1}^{\prime}, \ldots, \omega_{7}^{\prime}=\beta^{-1}, \frac{a}{c^{\prime} \epsilon}, \frac{b}{a^{\prime} \epsilon}, \frac{c}{b^{\prime} \epsilon}, \frac{c \epsilon}{a^{\prime}}, \frac{a \epsilon}{b^{\prime}}, \frac{b \epsilon}{c^{\prime}} .
\end{aligned}
$$

Let us set

$$
q=-\epsilon^{6}
$$

and $\frac{a}{b \epsilon^{2}}=-q, \frac{a^{\prime}}{b^{\prime} \epsilon^{2}}=-q^{-1}, c^{2}=c^{\prime 2}=a b=a^{\prime} b^{\prime}$. We get then

$$
\begin{aligned}
& \omega_{1} \ldots, \omega_{7}=\beta^{-1}, \epsilon^{-3}, \epsilon^{3}, \epsilon^{3}, \epsilon^{-3}, \epsilon^{-3}, \epsilon^{3} \\
& \omega_{1}^{\prime} \ldots, \omega_{7}^{\prime}=\beta^{-1}, \epsilon^{3}, \epsilon^{-3}, \epsilon^{-3}, \epsilon^{3}, \epsilon^{3}, \epsilon^{-3} .
\end{aligned}
$$

Let us now stick two such three-leg vertices together. The rules of the model completely determine the state of the intermediate edge from the ones of the outer edges, so we can as well forget it to get a standard four-leg vertex, whose weights determine a $\check{R}$-matrix. This matrix has two eigenvalues: 0 with degeneracy 6 , and $\beta^{-2}-q-q^{-1}$ with degeneracy 
three. The respective eigenvectors read

$$
\begin{aligned}
& |1,1\rangle \\
& |1,0\rangle+q|0,1\rangle \\
& |1,-1\rangle+q|-1,1\rangle ;|1,-1\rangle-q^{-1}|-1,1\rangle-\frac{a \epsilon \beta}{b^{\prime}}\left(1+q^{-2}\right)|0,0\rangle \\
& |-1,0\rangle+q|0,-1\rangle \\
& |-1,-1\rangle
\end{aligned}
$$

and

$$
\begin{aligned}
& |10\rangle-q^{-1}|0,1\rangle \\
& |1,-1\rangle-q^{-1}|-1,1\rangle-\frac{a \epsilon}{\beta b^{\prime}} q^{-1}|0,0\rangle \\
& |0,-1\rangle-q|-1,0\rangle
\end{aligned}
$$

Clearly this $\check{R}$-matrix has a "partial" $U_{q} s l(2)$ symmetry, where the generators $S^{ \pm}$only act on the states $| \pm 1\rangle$ as in a spin one-half representation (acting on $|0\rangle$ they just vanish). In the following we set $S^{z}= \pm 1,0$. The symmetry $U_{q} s l(2)$ therefore acts on the loop degrees of freedom without seeing the empty spaces in the system. By usual coproduct applications, this symmetry extends to the transfer matrix, up to boundary effects. Let us emphasize that the $O(n)$ vertex model has more symmetry than what we are pointing out here. In particular, at the solvable point of [32] there is a $U_{q^{\prime}} s l(2)$ symmetry acting on a full spin-one representation, with $q^{\prime}=\left(-\epsilon^{6}\right)^{1 / 4}$. In addition, in the low-temperature limit $\beta \rightarrow \infty$ there appears a $U_{q^{\prime \prime}} s l(3)$ symmetry [33] with $q^{\prime \prime}=\left(-\epsilon^{6}\right)^{1 / 2}$. Notice the equality

$$
n=-q-q^{-1}
$$

We restrict in what follows to the case $n \in[-2,2]$ for which $q$ is a complex number of modulus one.

Let us now discuss boundary conditions. As is well known, the correspondence between the vertex and the $O(n)$ models breaks down in a non-trivial topology due to noncontractible loops. For instance, on a cylinder non-contractible loops in the space direction have a weight 2 in the vertex model because they have the same number of left and right turns. This can be repaired by changing the vertex-model boundary conditions. Such an operation is also called putting a charge at infinity, or a seam [34]. Draw on the cylinder a line in the time direction. Give a vertex configuration an additional weight $e^{ \pm i \pi e}$ every time an oriented edge crosses the line going upwards (downwards) ( $e$ is well defined modulo 
two). In that case a non-contractible loop in space direction gets a weight $2 \cos \pi e$ which can be made equal to $n$ by the choice $e=e_{0}$. On a torus there are non-contractible loops with a more complicated topology. To reproduce their correct weight one has to consider a family of sectors of the $O(n)$ vertex model characterized by their "magnetic charge" $m=S^{z}$ (a topological defect $S^{z} \pi$ ) and the "electric charge" $e$ (a twist term) [35,36]. The sector with $-e$ and $-S^{z}$ has identical properties. Let us parametrize

$$
n=-2 \cos \pi \gamma, q=e^{i \pi \gamma}, \gamma \in[1,2]
$$

so in particular $\gamma=\left(e_{0}+1\right) \bmod 2$. The various sectors of the vertex model are then connected by quantum-group commutative diagrams [17]. This means that for $k$ greater than $S^{z}=m$, and $k-m$ integer, all levels of the sector $S^{z}=k, e=m \gamma$ are contained in the those of the sector $S^{z}=m, e=k \gamma$.

\subsection{The critical point}

The $O(n)$ model is critical when [7]

$$
\beta_{c}=(2+\sqrt{2-n})^{-1 / 2}
$$

Introduce solid-on-solid height variables $\phi$ dual to the arrows, with step value $\pm \pi$. In the continuum, these degrees of freedom become a free bosonic field with coupling constant, in the scale where topological defects are not renormalized,

$$
g=\gamma
$$

The above parameters $e, m$ are now interpreted as the usual electric and magnetic charges

in a two-dimensional Coulomb gas, with associated conformal weights $h_{e m}\left(\bar{h}_{e m}\right)=\frac{1}{4}\left(\frac{e}{\sqrt{g}} \pm\right.$ $m \sqrt{g})^{2}$. The partition function of the vertex model in the $S^{z}=m, e$ sector, and on a torus of size $R \times T$ reads in the continuum

$$
\mathcal{Z}_{e m} \rightarrow Z_{e m}=e^{f R} \frac{1}{\eta(y) \eta(\bar{y})} \sum_{j \in Z} y^{h_{e+2 j, m}} \bar{y}^{\bar{h}_{e+2 j, m}}
$$

for the simple geometry we consider, $y=\exp (-\pi T / R)=\bar{y}$. The central charge of this vertex model is equal to one for all values of $n$.

The aforementioned coincidences of levels are easily observed in (3.13). For example, the ground state of the sector $S^{z}=m, e=0$ has weight $h=\bar{h}=\frac{g m^{2}}{4}$, while weights in the 
sector $S^{z}=0, e=m \gamma$ are $h=\bar{h}=\frac{(g m+2 j)^{2}}{4 g}$, coinciding with the above for $j=0$. Such coincidences are well known to occur in the continuum limit as following from duality in the Gaussian model. We stress that they in fact occur for the finite lattice model, and for all values of $\beta$. Notice that $j=0$ is not necessarily the ground state of this sector: when $m=1$ we have $|2-g m|<g$ so that $j=-1$ is the ground state for $g>1$.

The partition function of the $O(n)$ model is obtained by proper summation of (3.13) for various sectors. An important fact is that the ground state of the $O(n)$ model is not the one of the vertex model. Choosing $e=e_{0}$ indeed gives the central charge

$$
c=1-\frac{6(g-1)^{2}}{g} \text {. }
$$

In conformal language, the $O(n)$ model is thus described by a twisted Gaussian model. In the $O(n)$ point of view, the value of $c=1$ for the untwisted Gaussian theory is a $c_{\text {eff }}$ value associated with the presence of a negative-dimension operator.

When $q$ is a root of unity, the $U_{q} s l(2)$ symmetry implies that additional levels coincide. We restrict for simplicity to the case

$$
n=2 \cos \frac{\pi}{t}, t=1,2, \ldots
$$

and restrict also to sectors $S^{z}=m$ an integer, where there are an even number of noncontractible loops in the space direction. In this case the commutative diagram of [17] extends further. Levels in the sector $S^{z}=m, e=k \gamma$ (with $k \geq m$ ) that are not in any other sector are selected by the restricted partition function

$$
\mathcal{Z}_{a b}=\sum_{j \in Z} \mathcal{Z}_{e=k \gamma, m+j t}-\mathcal{Z}_{e=m \gamma, k+j t}
$$

where

$$
a=k-S^{z}, b=k+S^{z}
$$

Differences like (3.16) are exact for finite lattices, due to the $U_{q} s l(2)$ symmetry.

Notice that the central charge of the $O(n)$ model reads in the parametrization (3.15)

$$
c=1-\frac{6}{t(t+1)}
$$

The $O(n)$ model at integer $t$ is not the same as the $t^{\text {th }}$ minimal model except for $t=2,3$ $(n=0,1)$ [37]. However, in the continuum limit the above restricted partition functions, 
as discussed in [17] for a similar case (Potts model), are appropriate pieces of the minimalmodel partition functions 2

$$
\mathcal{Z}_{a b}+\mathcal{Z}_{a+1 / \gamma, b+1 / \gamma} \rightarrow Z_{a b}=\sum_{s=1}^{t} \chi_{a s} \bar{\chi}_{b s}
$$

for $1 \leq a, b \leq t-1$, where $\chi$ are the usual characters of the corresponding irreducible representations of the Virasoro algebra for central charge (3.17), and the non-universal bulk free energy $f$ has been discarded. The above expressions sum up to the ones worked out in [36] to express the various minimal-model partition functions as linear combinations of Gaussian ones. For example, the diagonal (i.e. A-series) partition function reads

$$
Z_{A A}=Z_{c}\left[\frac{t+1}{t}, t\right]-Z_{c}\left[\frac{t+1}{t}, 1\right]
$$

where $Z_{c}$ is the Gaussian partition function in the notation of [36]. The expression for finite lattices analogous to (3.19) also makes sense due to the $U_{q} \operatorname{sl}(2)$ symmetry. Since this symmetry holds in fact for any $\beta$, all these expressions also make sense off the critical point.

\subsection{Massless flow}

As discovered in [7] the $O(n)$ model is also critical for $\beta>\beta_{c}$, with a continuum limit that is $\beta$-independent. In other words, this low-temperature phase is a flow to another fixed point, which turns out to also be a Gaussian model. This phase is often referred to as the "dense phase" by reference to polymers $(n=0)$. Of course the associated massless flow should be precisely the twisted version of the one discussed in section 2 . In particular since the $U_{q} \operatorname{sl}(2)$ symmetry is present all along the flow, and coincides in the UV and IR with the Gaussian model duality, the relations between $n, g$ and $\gamma$ valid at $\beta=\beta_{c}$ should still hold true. This does not require $g$ at the low-temperature fixed-point to be the same as in the massive phase; as we saw in section 2, we expect the coupling to get smaller. Thus another solution of these relations is applicable [7]; we have exactly

$$
g=2-\gamma=1-\frac{1}{t}
$$

${ }^{2}$ It is necessary to add sectors with $k=1 / \gamma$ to restrict the overall number of non-contractible loops to be even. 
The corresponding central charge is given again by (3.14) which reads here

$$
c=1-\frac{6}{t(t-1)}
$$

After proper restriction [12, 13, 15, this flow gives rise to the massless flow from the minimal model at central charge (3.17) to the one with (3.21). From the lattice point of view, as emphasized already, all coincidences of levels in the vertex model transfer matrix hold for every value of $\beta$. The sectors corresponding to the $O(n)$ model or the minimal models can be extracted by appropriate combinations of vertex partition functions. One checks easily that in the dense phase

$$
\mathcal{Z}_{a b}+\mathcal{Z}_{a+1 / \gamma, b+1 / \gamma} \rightarrow Z_{a b}=\sum_{r=1}^{t-2} \chi_{r a} \bar{\chi}_{r b}
$$

so we can see the flow of various sectors of the minimal models:

$$
\sum_{s=1}^{t} \chi_{a s} \bar{\chi}_{b s} \quad \text { flows to } \quad \sum_{r=1}^{t-2} \chi_{r a} \bar{\chi}_{r b}
$$

Such a result implies in particular that minimal models flow to a model of the same kind in the $A D E$ classification [38].

\subsection{Symmetries in the continuum}

We notice that the above $U_{q} s l(2)$ symmetry involves the same deformation parameter

as the $U_{q} \widehat{s l(2)}$ symmetry exhibited in the continuum theory (2.5) since the formal proof for the existence of this symmetry [15] does not depend on the sign of $\delta \beta$. We also recall that the soliton $S$-matrix describing the massive flow exhibits this symmetry as well. To a large extent one can formally identify the soliton trajectories with the loops of the underlying lattice model thanks to (3.9) [18, 19].

In the following we will use results for the continuum as well as the discrete theory, and usually refer to results in the latter case with an upper index " $l$ ".

\section{General numerical analysis: analytic continuation of massive TBA and lattice data}

\subsection{Analytic continuation}

A natural way of getting insight into the phase structure of the $O(n)$ model is to determine its ground-state energy $E_{0}(R)$, where space is a cylinder of circumference $R$. 
There are two methods we will use to find this quantity. The first is to explicitly diagonalize the lattice hamiltonian (or transfer matrix - we shall refer to these two interchangeably) on a small, periodic lattice. The second way relies on the fact that when spacetime is a cylinder of radius $R$ and a large length $T$, the partition function is $Z \sim \exp \left(-E_{0}(R) T\right)$. If we reverse the roles of space and time, this is the partition function for a large system at temperature $1 / R$.

If the $O(n)$ lattice model were integrable for $\beta^{l} \neq \beta_{c}^{l}$ one could use the lattice Bethe ansatz to diagonalize the Hamiltonian for a large number of sites and then do the thermodynamics using the resulting equations for the energy levels [39]. Unfortunately, this does not seem possible here. However, this model is integrable in the continuum. Thus we can use a related but different method, called the thermodynamic Bethe ansatz (TBA) [40]. Here one conjectures the exact $S$-matrix for the particles of the theory, and uses the relation between the $S$-matrix and the phase shifts of the wave function in order to find the equations for the energy levels. Like in the ordinary Bethe ansatz case, one then uses these energy levels to minimize the free energy. The $S$-matrix for the sine-Gordon model (2.5) with $\delta \beta>0$ has been known for a long time [25], and the TBA equations were written in [21]. As we discuss in the sequel, there are complications in understanding possible $S$-matrices and the TBA for $\delta \beta<0$. It is thus useful to consider first analytic continuation of these high-temperature results to the massless phase. In this subsection we do this numerically; there seems to be no way of continuing the TBA equations directly. In [3] a similar procedure essentially reproduced the flow for the ground state energy from the tricritical to the critical Ising model, a result which was then confirmed by using a "massless $S$-matrix".

Our results can be written in terms of the dimensionless quantity $m R$, where $m \propto$ $(\delta \beta)^{(t+1) / 4}$. (The coefficient of proportionality can be obtained by comparing the results of conformal perturbation theory and the TBA. Similarly, on the lattice $m \propto\left(\delta \beta^{l}\right)^{(t+1) / 4}$, where the coefficient is determined by numerical study.) We define the quantity $c(m R) \equiv$ $6 R E_{0}(R) / \pi$, to take advantage of the fact that in unitary theories $c(0)$ and $c(\infty)$ are the central charges of the UV and IR conformal field theories [34,41]. We note that off the critical points this is not the $c$-function defined in [1], but at lowest order in $\delta \beta$ the two are the same. It is expected (and has been shown in all known unitary cases) to decrease monotonically as $m R$ is increased. In non-unitary theories $c(0)$ and $c(\infty)$ are not the central charges but rather $c_{\text {eff }} \equiv c-12 h-12 \bar{h}$, where $h$ and $\bar{h}$ are the lowest conformal dimensions in the sector [34,41]. Efforts to find a $c_{\mathrm{eff}}$-theorem have used this observation 
as a starting point. This makes the ground-state energy a logical candidate, but as we saw in section 2 , it does not always decrease!

The TBA gives a set of integral equations which allow the determination of $c(m R)$ in the high-temperature phase. They have not been solved directly, but it is straightforward to solve them numerically. There exists an expansion around $m R=0$

$$
c(z)=c_{U V}+c_{\mathrm{bulk}}(z)+\sum_{n} f_{n} z^{n}
$$

where we have defined $z=(m R)^{4 /(t+1)}$. We also use the variable $r=m R$. This corresponds to the perturbative expansion of (2.5) in powers of $\delta \beta$ (because of the $\mathbf{Z}_{2}$ symmetry $\phi \rightarrow-\phi$, there are only even powers of $(\delta \beta)^{1 / 2}$ in the expansion). The non-universal bulk term does not appear in perturbed conformal field theory and so is not necessarily an integer power of $z$. For $t$ integer this bulk term is the same for the full sine-Gordon theory and for the minimal model, where it was computed in [3]. This is because the models differ by boundary conditions only; the truncation does not affect bulk properties. More precisely one can check numerically (and analytically in related models like the 8-vertex model) that in the IR the levels of the vertex-model transfer matrix that belong to the minimal model are still at finite distance in the $1 / R$ scale from its ground state, implying that all these levels share the same bulk term.

To do the analytic continuation, we first solve the TBA equations for $c$ in the massive phase to double-precision accuracy. We then subtract off the massive bulk term. For $t$ even, this term vanishes, but for $t$ odd it is proportional to $(m R)^{2} \ln (m R)$ [3]. After subtraction, we have a power series in $z$ which can be continued to negative values. We do this in one of two ways, both involving Padé extrapolation.

The first method is to find the ratio of polynomials which go though a set of $z \geq 0$ data points $\left(z_{i}, c\left(z_{i}\right)\right)$ determined numerically using the TBA integral equations. The ratio is unique after one specifies the orders of the numerator and the denominator; their sum is the number of data points plus one. Typically, the most accurate results are obtained by using 10-15 data points. We have used the routine in Numerical Recipes [42]. It is particularly useful because it provides a convenient way of estimating the error. By using different subsets of the original data points, one obtains different extrapolations. The error is then estimated by looking at how the result changes for a given value of $z$.

The second method is to find the coefficients $f_{n}$ in (4.1) by fitting them to the numerically-determined $c(m R)$ function, using a standard least-mean-squares program. 
One then analytically (it is convenient to use Maple or Mathematica) finds the Padé approximant. (For a polynomial of order $p$, the Padé approximant is the unique ratio of polynomials of order $p / 2$ such that when this is expanded around $z=0$, the first $p+1$ terms reproduce the original polynomial. If $p$ is odd, then the polynomial in the numerator is of order $(p+1) / 2$ with the denominator of order $(p-1) / 2)$. The Padé approximant should describe $c$ for negative $z$ for much larger values of $|z|$ than does the polynomial. This method has the disadvantage that it is it is not as easy to determine the errors involved, and it requires more computer time, because many more data points are needed for a good fit than for the first method. For example, in the $t=2(n=0)$ case, one finds

$$
c(z)=1 .-.4454536 z+.091380 z^{2}-.00903 z^{3}-.00018 z^{4}+.00015 z^{5},
$$

where the numerical error is in the last digit given. The resulting Padé approximant is

$$
c(z)=\frac{1-.301756 z+.0436 z^{2}-.00314 z^{3}}{1+.143698 z+.0162 z^{2}} .
$$

We have checked for several values of $t$ that the results obtained by these two methods are consistent, but we have mainly used the first method.

It is easy to find the first extremum of the $c$-function using this continuation. For large $t$ this allows us to check formula (2.17) for this maximum, which is determined by quite a different route. Before making the comparison, we must subtract off the low-temperature bulk term. The situation here is a little more complicated than for the massive flow. For $0<n \leq 2$ the bulk term is the same for the full sine-Gordon model and the minimal theory, and we can use the results of [3]. This is not true for $n=0(t=2)$ due to a large number of level crossings which we shall discuss further. For the sine-Gordon bulk term, our results are still consistent with putting $t=2$ in the minimal-model formulas. The agreement is very good, as exemplified in the following table that gives the ratio of $c_{\max }-1$ for the continuation and (2.17)

$\begin{array}{ccccccc}\mathrm{t}=2 & \mathrm{t}=3 & \mathrm{t}=4 & \mathrm{t}=5 & \mathrm{t}=6 & \mathrm{t}=7 & \mathrm{t}=8 \\ .556 & .685 & .759 & .806 & .840 & .87 & .88\end{array}$

We certainly see the $1 / t^{3}$ behavior of the maximum being reproduced in the continuation, and standard extrapolation as a function of $1 / t$ shows very good convergence to the value one. As $t$ increases the number of coupled equations in the massive TBA increases, making the calculation more difficult, as well as the effect smaller to see. Other features of the 
large- $t$ expansion can also be checked against the TBA results, like the ratio of the $r$ values at the maximum and at the intersection with $c=1$.

We have not been able to extend the continuation far enough to observe a minimum, nor $c$ going back to one in the IR. We give an example of data obtained in figure 1 for $n=\sqrt{2}$.

It is difficult to determine precisely the radius of convergence of the $z$ expansion for $c(m R)$, but several tests provide a value around $z_{c} \approx 1$. The Padé extrapolation typically gives reliable data up to $z \approx 3$ for $n=0$, and less as $n$ increases. Singularities in the complex plane can be localized using the Padé approximants, but their position depends too much on the number of points used to be reliable. There was however no sign of these singularities getting too close to the real axis, negative or positive. (If there were a pole on the negative axis, the terms in (4.2) should alternate in sign.)

\subsection{Lattice results}

A complementary tool for studying the massless flow is the numerical diagonalization of the $O(n)$ vertex model transfer matrix on cylinders of lattice width $R^{l}$. (Since we will use results for the continuum as well as the discrete theory, we often refer to results in the latter case with an upper index "l".) This provides lattice data that, after proper extrapolation $\left|\beta^{l}-\beta_{c}^{l}\right| \rightarrow 0, R^{l} \rightarrow \infty$ with $r^{l} \equiv\left|\beta^{l}-\beta_{c}^{l}\right|^{\nu} R^{l}$ fixed, should reproduce the continuum limit of interest $\left(r\right.$ and $r^{l}$ being related by a non-universal proportionality constant). A difficulty here is to extract the bulk term. Several obvious strategies are possible, none of them giving too-satisfactory results. Their comparison however allows the determination of $c\left(r^{l}\right)$ with a reasonable accuracy, generally better for small or large values of $r^{l}$ and for $t$ large. A convenient number to consider is

$$
c^{\left(R^{l}\right)}\left(r^{l}\right)=\frac{\frac{\log \Lambda^{\left(R_{1}^{l}\right)}\left(m^{l}\right)}{R_{1}^{l}}-\frac{\log \Lambda^{\left(R_{2}^{l}\right)}\left(m^{l}\right)}{R_{2}^{l}}}{\frac{1}{\left(R_{1}^{l}\right)^{2}}-\frac{1}{\left(R_{2}^{l}\right)^{2}}} .
$$

where $R_{1}^{l}$ and $R_{2}^{l}$ are neighboring widths (eg $R^{l}, R^{l}-1$ ). In the study of critical points such combination is known to converge very rapidly to the exact value $c$, with the bulk term conveniently extracted. In our situation, we are not allowed a priori to use this procedure, since apart from a regular term, the bulk term (actually its singular part from the lattice point of view, but that is all what is seen in the continuum limit) also scales like $1 / R^{l}$ in the limit we are interested in. On the other hand, corrections to scaling for finite $R^{l}$ seem 
to compensate conveniently so that (4.4) is a rather reliable estimate, especially at $t$ large, i.e. it coincides with estimates obtained by other, more correct means, up to error bars. As an example we give also in figure 1 the result obtained from (4.4) for $n=\sqrt{2}$. After proper rescaling of the lattice and continuum variables, it is seen to nicely coincide with the result from TBA extrapolation in the region where they are reliable. For another example we

give in the following table the maximum of the function of $c^{\left(R^{l}\right)}\left(\right.$ for $R_{1}^{l}=R^{l}, R_{2}^{l}=R^{l}-1$ ) for various sizes

$\begin{array}{llllll}R^{l}= & 4 & 5 & 6 & 7 & 8 \\ c_{\max }= & 1.024 & 1.017 & 1.016 & 1.015 & 1.015\end{array}$

which seem to be converging nicely to the TBA value $c_{\max }=1.0137$. In the lattice simulation (but not the TBA) one observes further $c$ reaching a minima, and going back to the value 1 in the IR. Details of such curves can be checked against the large $t$ results, like the value of $c$ at the minima, the ratio between the values of $r^{l}$ at the maxima and minima, or at the maxima and the point $c=1$, with very reasonable agreement.

Two additional features must be mentioned. First we have observed a general tendency for the TBA and lattice data to differ after some value of $r^{l}$, at the border of the domain where the TBA data are reliable. This is observed in particular for the unsubtracted case, where lattice data are affected only by the small uncertainties of the $R^{l} \rightarrow \infty$ extrapolation. In addition, we have observed a large unstability of the lattice data in the region $r^{l}$ large. This is very marked for $n<0$ in particular, where for $R^{l}$ large enough we have observed another pair of extrema in (4.4) as well as related features in the unsubtracted data. An example is given for $n=-1$ in figure 2 .

\section{The boundary case $n=-2$}

The case $n=-2$ is easily solvable. Indeed, the associated Gaussian model at the UV fixed point has $g=2$, and in the Thirring version, the four-fermion coupling vanishes. This is actually the "natural" boundary of the behavior we want to study since the dense $O(n=-2)$ model, corresponding formally to $g=0$ in $(3.20)$, is in fact massive. So we shall not be able to recover a Gaussian model in the IR, and this example is pathological in some respects. 
That we are dealing with free fermions can also be seen directly from the point of view of the $O(n=-2)$ lattice model. Consider indeed the fermionic integral

$$
\mathcal{Z}=\int d \psi d \psi^{+} \prod_{i} e^{-\psi_{i} \psi_{i}^{+}} \prod_{\langle j k\rangle} \exp \left[\beta^{l}\left(\psi_{j}^{+} \psi_{k}+\psi_{k}^{+} \psi_{j}\right)\right]
$$

By usual rules of Grassman integration this gives rise to an expansion similar to (3.1) with the only difference that "loops" covering a single edge (corresponding to terms like $\left.\psi_{j}^{+} \psi_{k} \psi_{k}^{+} \psi_{j}\right)$ are allowed here. Such a model of loops + dimers [43] is expected to be in the same universality class as (3.1). On the other hand it is exactly solvable by elementary techniques since (5.1) is equal to the determinant of the interaction matrix. The critical point occurs at $\beta_{c}^{l}=1 / 3$ where this matrix restricts to the discrete Laplacian. For $\beta$ close to $\beta_{c}$ (mapping between lattice and continuum is straightforward here, so we suppress the lattice label) the matrix coincides with $\Delta+m^{2}$ with $m^{2}=9\left(\beta_{c}-\beta\right)$. Therefore the massive flow corresponds to a positive $m^{2}$ while the massless flow corresponds to negative $m^{2}$. These results are in agreement with the value $h=\bar{h}=0$ for the thermal operator of the $O(n=-2)$ model. The sector where non-contractible loops in the spatial direction have weight 2 is reproduced by choosing antiperiodic boundary conditions for the fermions on the cylinder. In the massive flow the running central charge is immediately obtained from [44,45]:

$$
\begin{aligned}
c=1 & -\frac{3 r^{2}}{\pi^{2}}\left(\ln \frac{\pi}{r}+\frac{1}{2}+-\gamma_{E}\right) \\
& +\frac{12}{\pi} \sum_{k=1}^{\infty}\left[\sqrt{(2 k-1)^{2} \pi^{2}+r^{2}}-(2 k-1) \pi-\frac{r^{2}}{2(2 k-1) \pi}\right],
\end{aligned}
$$

where $r=m R$. In the standard $O(n=-2)$ model this result is still expected to hold by universality, although the correspondence between the mass $m$ and the lattice temperature may involve additional logarithmic contributions.

Consider now the massless direction. The above formula is still expected to hold, up to a bulk $r^{2}$ (and possible logarithmic corrections) term. The striking fact is then the presence of the square root singularities which, beyond the value $r^{2}=-(2 k-1)^{2} \pi^{2}$, develop an imaginary part. (Similar square-root singularites seem to appear in affine Toda theories at negative $z$ [45]). Recalling the expression for the continuum partition function 
(including massive states as well as the ground state) of the $O(n=-2)$ model in that particular sector, where $T$ is the length of the cylinder,

$$
\exp \left(4 \pi \frac{T}{R} \frac{c}{24}\right)\left\{\prod_{k}\left[1+\exp \left(-\frac{T}{R} \sqrt{(2 k-1)^{2} \pi^{2}+r^{2}}\right)\right]^{2}+(-)^{2}\right\}
$$

we see for instance beyond $r^{2}=-\pi^{2}$ the two largest eigenvalues become equal in modulus. Successive branchings occur as $r^{2}$ crosses the roots $-(2 k-1)^{2} \pi^{2}$. It is not clear how to define a central charge when the largest eigenvalue becomes complex. The most natural candidate is the real part of (5.2) (the modulus of the largest eigenvalue). Because of the square-root term, it is singular with an infinite derivative at each of the points $r^{2}=$ $-(2 k-1)^{2} \pi^{2}$, and shows wide oscillations moving away from the UV fixed point. This is fully confirmed by the numerical study of the lattice $O(n=-2)$ model (3.1).

It is interesting to compare this situation with the square-lattice Ising model. Introducing the variable $v=\tanh \beta / 2$ the locus of zeroes of the partition function is a set of two circles depicted in figure 3. The ferromagnetic critical point is the intersection of the rightmost circle with the positive real axis. Away from the critical point the Ising partition function is a sum of square roots of determinants of the massive Laplacian. The case $m^{2}$ real and positive corresponds to moving on the real physical axis (in which direction depends on the sign of $m$ ). The case $m^{2}$ real and negative corresponds to moving exactly along the circle of singularities. The intersection of the two circles corresponds to $m^{2}=-4$ which is also the opposite of the maximum eigenvalue of the massless Laplacian on the square lattice.

That the largest eigenvalue of the transfer matrix in a sector can branch and become complex for real $\beta$ seems peculiar to $n=-2$ [46]. For any other $n \in[-2,2]$ and for $R^{l}$ large enough this eigenvalue appears to remain real in our computations. It must be so in the $r^{l} \rightarrow \infty$ limit so that it agrees with the known spectrum in the continuum.

Although pathological, the case $n=-2$ is interesting since then the running central charge presents an infinite sequence of singularities and oscillations. This is a strong indication that for $n$ negative and close enough to $n=-2$ similar oscillations are present (a fact we observed numerically above for $n=-1$ ) and maybe also some singularities. The region $n$ negative seems more difficult to study because of these features, and in the remainder of this paper and the sequel we mainly consider $0 \leq n \leq 2$.

As a final comment, we note that the general case should also be amenable by exact solution (using the Bethe ansatz) of the Thirring model with imaginary mass. We have not done this calculation yet. 


\section{Detailed study of the case $n=0$ : Spontaneous breaking of $N=2$ supersymmetry}

We now discuss in some detail the case $n=0$. It is closely related to the physics of selfavoiding walks and also to $N=2$ supersymmetry [47]. Again, we analyze the ground-state energy. It was shown in [48 that the sine-Gordon model (2.5) is $N=2$ supersymmetric at the coupling where $n=0$. This will prove a very powerful tool. The fermion number $F$ in the $N=2$ language is equal to half the soliton number in the massive sine-Gordon model.

The results depend crucially on the boundary conditions around the cylinder [21]. These are implemented by giving non-contractible loops a weight $2 \cos \pi e$ in the $O(n)$ model, which corresponds to a fugacity for the sine-Gordon solitons of exp $\pm i e \pi$. In the $N=2$ picture, this corresponds to inserting the operator $e^{i 2 \pi e F}$. The boundary conditions used in sect. 4 have $e=0$, and in the $N=2$ language these are called Neveu-Schwarz. These give an effective central charge $c=1$ in the UV limit. We first discuss the situation with twisted boundary conditions, which have a "charge at infinity" $e=e_{0}= \pm 1 / 2$. This gives non-contractible loops a weight of zero, and has $c_{U V}=0$. In the $N=2$ picture this means that we insert $(-)^{F}$, and is called the Ramond sector. These boundary conditions have the advantage that we can derive quantitative information by using the supersymmetry, even in the massless phase.

\subsection{The Ramond sector}

a) Preliminary: one-dimensional phase transitions for polymers

There are several aspects that deserve attention. As a preliminary we recall that for lattice cylinders of finite circumference $R^{l}$, the flow to the dense phase is characterized by an infinite sequence of "first-order" phase transitions, taking place at values of $\beta^{l}>\beta_{c}^{l}$ which depend on $R^{l}$. These transitions are easily understood by studying the correlation functions of $L$-leg operators, which are defined by "forcing" a set of $L$ lines to propagate on a cylinder (see figure 4 ) with as usual a weight $\beta^{l}$ per edge. These operators have UV conformal dimensions $h=\bar{h}=\left(9 L^{2}-4\right) / 96$. The resulting partition function grows like the largest eigenvalue $\Lambda_{L}^{\left(R^{l}\right)}$ of the corresponding transfer matrix. For $\beta^{l} \leq \beta_{c}^{l}$ self-avoiding walks cover a negligible fraction of the lattice, so this eigenvalue must be smaller than one. If the total number of possible configurations for this set of $L$ lines of total number of edges $\mathcal{N}$ grows as $\Omega_{L}^{\left(R^{l}\right)} \propto\left(\mu_{L}^{\left(R^{l}\right)}\right)^{\mathcal{N}}$, they will start occupying a finite fraction of available space when $\beta^{l}=\frac{1}{\mu_{L}^{\left(R^{l}\right)}}$. In other words, this eigenvalue of the transfer matrix will cross 
the previously-dominant trivial eigenvalue, which is always equal to one for any $\beta^{l}$. The polymer density is discontinuous at that point, and this crossing can be described as a first-order one-dimensional phase transition [49].

To illustrate this point, it is clear that the transfer matrix for propagation of two (unoriented) lines on a cylinder of radius $R^{l}=2$ is one-by-one, with a single eigenvalue equal to $2\left(\beta^{l}\right)^{4}$. The latter crosses one for $\beta^{l}=2^{-1 / 4}$ [50].

For fixed $L, 1 / \mu_{L}^{\left(R^{l}\right)} \rightarrow \beta_{c}^{l}$ as $R^{l} \rightarrow \infty$. Moreover, by the usual finite-size scaling arguments, $\left(1 / \mu_{L}^{\left(R^{l}\right)}-\beta_{c}^{l}\right)^{\nu} \propto 1 / R^{l}$. Therefore in the "blown-up" variables of the continuum limit, the transitions occur at finite distance from one another. We introduce the notation

$$
\lim _{R^{l} \rightarrow \infty}\left(1 / \mu_{L}^{\left(R^{l}\right)}-\beta_{c}^{l}\right)^{\nu} R^{l}=r_{L}^{l}
$$

A set of estimates of $r_{L}^{l}$ obtained from lattice computations is given in the following table.

$\begin{array}{rllllll} & R^{l}=\mathrm{L} & R^{l}=\mathrm{L}+1 & R^{l}=\mathrm{L}+2 & R^{l}=\mathrm{L}+3 & R^{l}=\mathrm{L}+4 & \\ L=2 & .8101 & .779 & .759 & .746 & .738 \longrightarrow & .725 \\ 4 & 1.920 & 1.788 & 1.725 & 1.681 & 1.653 \longrightarrow & 1.6 \\ 6 & 3.032 & 2.811 & 2.700 & 2.63 & 2.55 \longrightarrow 2.2 \\ 8 & 4.147 & 3.85 & & & & \end{array}$

where the last column is the extrapolation to $R^{l} \rightarrow \infty$. They are universal up to an overall scale.

b) Level crossing in the Ramond sector

By using the quantum-group symmetries discussed in sect. 3, one easily finds that the eigenvalues describing $L$-leg operators are also present in the Ramond sector $(e=1 / 2)$ for $L=2 \bmod 4$. One can see this explicitly for the value $R^{l}=2$, where the transfer matrix of the $O(n=0)$ vertex model in the sector of vanishing spin reads

$$
\left(\begin{array}{ccc}
\left(\beta^{l}\right)^{4} & -i\left(\beta^{l}\right)^{4} & 0 \\
i\left(\beta^{l}\right)^{4} & \left(\beta^{l}\right)^{4} & 0 \\
2\left(\beta^{l}\right)^{2} i^{1 / 2} & 2\left(\beta^{l}\right)^{2} i^{-1 / 2} & 1
\end{array}\right)
$$

The eigenvalues are $1,2\left(\beta^{l}\right)^{4}, 0$. Hence at $r_{2+4 n}^{l}$ a new eigenvalue crosses the value 1 , which of course is the continuation of the largest eigenvalue in the high-temperature phase. This crossing is a first-order phase transition for finite $R^{l}$ and remains so in the $R^{l} \rightarrow \infty$ limit, as can easily be checked using scaling or by numerical analysis. 
Much can be learned about these first-order phase transitions from the $N=2$ point of view. Well-known arguments [51] show that the Ramond ground-state energy being zero is equivalent to supersymmetry remaining unbroken (for unitary theories). Since the Witten index is not zero here, supersymmetry cannot be broken in the high-temperature phase where the model is unitary, and indeed the largest eigenvalue of the transfer matrix is one. However, unitarity is lost in the low-temperature phase, and it is possible for a level to cross zero energy (the continuation of the Ramond ground state) and become negative. This is actually easy to see using the $N=2$ Landau-Ginzburg action [52 with superpotential

$$
X^{3}+\bar{X}^{3}+(\delta \beta)^{1 / 2}(X+\bar{X})
$$

For $\beta>\beta_{c}$ the potential is not positive (or even real), ensuring that the ground-state energy does not have to be zero. We can say that supersymmetry is spontaneously broken in the Ramond sector for $r^{l}>r_{2}^{l}$, and that at each $r_{2+4 n}^{l}$ a new level crosses energy zero. Notice however that, since the perturbation does not break supersymmetry explicitly, the Witten index remains equal to its UV value, and in fact a pair of levels with opposite values of $(-)^{F}$ cross zero together.

If one simply measures the ground state energy of the $R$ sector, it is zero up to $r_{2}^{l}$ and then non-zero with a discontinuity of the first derivative at that point. However there is also a discontinuity of the bulk term, so if one measures running ground-state energies after subtracting this bulk term, the final result has a discontinuity at $r_{2}^{l}$, as is illustrated in figure 5 from lattice data. This is an indication that the analytic continuation done in the last section only describes the ground state energy up to $r_{2}^{l}$ : since $c=0$ in the Ramond sector for $z \geq 0$, analytic continuation obviously gives $c=0$ for all $z$. Of course all the above results apply without reference to the lattice model, and using the variable $r$.

This picture can be further elucidated by considering the "index" studied in [22, 23], $Q=i(R / T) \operatorname{tr} e^{-R H} F(-)^{F}$. 3 The index $Q$ is obtained by taking the derivative of the ground state energy with respect to $e$ at $e=1 / 2$. It has a simple polymer interpretation in the massive flow: it is the partition function for a single non-contractible loop [21]. This index has the advantage that we can analytically derive an differential equation for it even in the massless phase, as opposed to the ground-state energy in the Neveu-Schwarz sector discussed in the last section, where the TBA integral equations can only be numerically

3 Notice that this Hamiltonian propagates the system in the $R$-direction, as opposed the transfer matrix we studied above, which is in the $T$-direction. 
continued. (If one tries to continue the equations directly, the integrals diverge.) Since all of the derivations of [22] hold for any superpotential, they should hold even in the case of a non-real potential like (6.3) in the dense phase. It was shown in [22, 23] that in the massive phase $Q$ satisfies a differential equation of Painlevé III type: we have

$$
\frac{d^{2} U}{d r^{2}}+\frac{1}{r} \frac{d U}{d r}=\sinh U
$$

with

$$
Q=\frac{r}{2} \frac{d U}{d r}
$$

Near $r \equiv m R=0$ the solution we are interested in looks like

$$
U \sim \frac{2}{3} \log \frac{r}{2}+s+\sum_{n} g_{n} r^{(4 n / 3)},
$$

where $\exp (s / 2)=\Gamma(1 / 3) /\left(2^{2 / 3} \Gamma(2 / 3)\right)$ [53]. As before, we define the variable $z=r^{4 / 3}$. The low temperature phase $\beta>\beta_{c}$ corresponds to $z$ negative but real. Defining $u=U+i \pi / 2$, (6.4) is then

$$
\frac{d^{2} u}{d z^{2}}+\frac{1}{z} \frac{d u}{d z}=\frac{9}{16 \sqrt{-z}} \cosh u
$$

It follows from (6.6) that our solution for $u$ and $Q$ is real for negative $z$.

For $r$ near $r_{2+4 n}^{l}$ two eigenvalues of the transfer matrix (two levels of the hamiltonian) are very close for $e=1 / 2$, and are coupled if $e \sim 1 / 2$ by the standard formula of secondorder perturbation theory. It is easy to show that the numerator is of order $e-1 / 2$ while of course the denominator is the difference of energies of the two levels. Therefore we expect the derivative with respect to $e$ to exhibit a simple pole at $r_{2+4 n}$, that is we expect simple poles for $Q$. That $Q$ should become infinite when a level crosses zero energy is clear a priori from its definition where no bulk term contribution is subtracted. In terms of polymers this pole corresponds to the sudden growth of infinite arms to the non-contractible loop (figure $4 \mathrm{~b}$ ). This can be seen explicitly on the lattice for radius $R^{l}=2$, where for $e$ close to $1 / 2$ the eigenvalue that is dominant in the UV reads

$$
1+\left(2 i \beta^{4}-\frac{4 i \beta^{6}}{1-2 \beta^{4}}\right) \pi(e-1 / 2) .
$$

Hence $Q$ should exhibit an infinite sequence of simple poles in this massless flow. A numerical solution of (6.7) fully confirms this prediction. The locations of the first poles (in the variable $r$ ) are 


$\begin{array}{lllll}1^{\text {st }} & 2^{\text {nd }} & 3^{\text {rd }} & 4^{\text {th }} & 5^{\text {th }} \\ 2.95708396 & 8.61 & 14.1 & 19.5 & 24.8\end{array}$

Taking ratios to eliminate the lattice-dependent mass scale they agree well with the above results of lattice computations. For the reader with this particular interest we give independently in the appendix the results for polymers deduced from this study.

In fact, we can extract information about these poles analytically. The first pole $z_{1}$ can be determined from the coefficients $g_{n}$ in (6.6): as long as there are no other poles nearby, the coefficients should be the same as those of the expansion of $1 /\left(z-z_{1}\right)$ in powers of $z$. The $g_{n}$ can be found analytically by substituting (6.6) into (6.4); for example, $g_{1}=-(9 / 32) 2^{2 / 3} e^{-s}$. Alternatively, one can fit these coefficients to a ratio of polynomials as in sect. 4 , and find the first pole in this manner (this is why the location of this pole is known very accurately).

It is easy to show that all poles in $Q$ are simple ones. Near a pole, the cosh-Gordon equation (6.7) reduces to the (radial part) of the Liouville equation (i.e. $\cosh u \sim e^{u} / 2$ ). This can be solved explicitly; the general solution near a pole at $z=z_{0}$ is

$$
u(z) \sim \ln \frac{-64}{9 \sqrt{-z}} \frac{z_{0}}{\left(z-z_{0}\right)^{2}}
$$

Thus all poles in $Q \propto d u / d z$ are simple.

We can also see that there are an infinite number of poles on the negative $z$-axis. We show that given one pole, there is another one a finite distance farther along the axis. The crucial fact is that in any given region, the right-hand side of (6.7) is finite and positive. (It may appear more comfortable to suppress the $1 / \sqrt{-z}$ by switching to the variable $w=(-z)^{4 / 3}$, where this factor disappears and the right-hand side is $\geq 1$.) As we move away from the pole in the negative direction, $u^{\prime \prime}$ and $u^{\prime}$ are positive. As long as $u^{\prime} / z$ is negative, (6.7) requires that $u^{\prime \prime}$ is positive and finite. Thus $u^{\prime}$ must decrease at a finite rate and eventually hit zero. This is a minimum of $u$, because on the negative $z$-axis, $u$ has no maxima (only poles): if $u^{\prime}=0, u^{\prime \prime}>0$. Thus after the minimum, $u$ must start increasing and not turn around. It must increase at a finite rate, so eventually $u$ will get large enough so that we can use replace (6.7) with the Liouville equation, and our solution is of the form (6.9). Since $u^{\prime}$ is negative and must remain negative until we hit a pole, the pole in (6.9) must fall on the negative axis. Thus we have established that there is another pole a finite distance away at larger $|z|$. 
In fact, for $|z|$ large we can show that the distance between a pole at $z_{i}$ and the next pole is $\sim C\left|z_{i}\right|^{1 / 4}$, where $C$ is a constant which is about 7 . This is done by patching together the solution (6.9) for $u$ large with the solution for $u$ small (i.e. $\cosh u \sim 1$ ), which is

$$
c(z)=\frac{1}{4} z^{3 / 2}+A \ln z+B .
$$

As $r \rightarrow \infty$ an infinite number of levels crosses the value one. The Witten index $\operatorname{tr}(-1)^{F}$ is still equal to 2 . However, most levels other than the ones of vanishing energy have a bulk term that becomes infinite in that limit. If we subtract this bulk term, we have to set the Witten index to zero for consistency. This is in agreement with the study of the IR fixed point described by an $\eta \xi$ system. We have here two theories related by a $N=2$ preserving flow which have different Witten indices, so they must be considered "at infinite distance" from each other [54]. This sheds some doubt about the possibility of connecting them smoothly by a TBA-like description, an issue which we will discuss further in the next paper.

Besides the crossing of eigenvalues at $r_{2+4 n}^{l}$ just explained by quantum-group symmetries, we have observed additional crossings at values that tend toward $r_{4+4 n}^{l}$ for large lattice systems. The matrix elements are such that these crossings do not produce a pole in $Q$.

\subsection{The Neveu-Schwarz sector}

\section{a) Observations}

This is the sector discussed in sect. 4, where solitons for the massive flow in the sineGordon model have fugacity one, and non-contractible loops in the $O(n)$ vertex model are given a weight 2. Unfortunately, there is no index in this sector and the TBA continuation of sect. 4 breaks down after the first maximum. We here discuss only the lattice results. As in the large- $t$ expansion, $c=1$ is observed both in the UV and IR. A remarkable feature, very different from the massive study, is the very slow stabilization of the curve's shape as the lattice size is increased, and one may wonder if this hides further aspects, unobservable at the sizes we can study. However, a clear result which generalizes to all values of $n>-2$ is that the largest eigenvalue of the transfer matrix remains real and non-degenerate at all values of $r^{l}$. For the moment we start by doing some "numerology". Comparison of estimates for $r_{L}^{l}$ with figure 6 shows that peaks of the derivative $\frac{d^{2} c}{d\left(r^{l}\right)^{2}}$ occur roughly at $r_{2}^{l}$ and $r_{4}^{l}$. These coincidences are confirmed by a more detailed study, and they hold 
within our error bars. At $r^{l}=r_{2}^{l}$ there occured a phase transition in the Ramond sector, associated with a discontinuity of the running effective central charge. It is not surprising that $r_{2}^{l}$ emerges as well in the study of the $N S$ sector. Even though we are a finite distance from the UV fixed point in the blown-up variable $r^{l}$, we are still infinitesimally close to it, and the polymers present in the ground state of this sector still occupy an infinitesimally small fraction of the available space. This does not change the fact that at $r_{2}^{l}$ two-leg diagrams become infinite. One may actually wonder whether $r_{2}^{l}$ (as well as $r_{2 n}^{l}$ ) could be a point of phase transition in the sine-Gordon model itself, independent of the sector; we shall discuss this further.

We have also observed that the two (first) extrema of the curve $c\left(r^{l}\right)$ occur roughly at the values $r_{1}^{l}$ and $r_{3}^{l}$. Study of the present data indicates that they do not exactly take place there, although as always this may be a finite-size effect. We do not expect any special feature to occur in the Ramond or Neveu-Schwarz sector at values $r_{1+2 n}^{l}$. This is because the transfer matrix we are considering acts on spin zero in the vertex model language, so only even numbers of lines can propagate through the system.

b) Possible singularities

The evidence seems to exclude a discontinuity in $c(m R)$. As far as its derivatives are concerned, they are difficult to study numerically. The lattice-subtracted quantity (4.4) is not very reliable for determining derivatives, nor are any of the procedures we have used to subtract the bulk term. We can of course study derivatives of unsubtracted quantities, but then their convergence as $L$ increases is affected by $c$ and the bulk term, and this mixture is difficult to disentangle. The safest quantity to study seems to be the second derivative with respect to $r$ of the full ground-state energy, including the bulk contribution. In the large $R^{l}$ limit, the bulk term should simply contribute an additive constant. Results are shown in figure 7 . There is a spectacular peak at values that converge to $r_{2}^{l}$. The convergence past this value is however quite slow, and makes the quantitative analysis of this peak difficult. We have done the same analysis for the Ramond sector, where we know that we should observe a delta function. The numerical results look virtually identical to these past $r_{2}^{l}$. We therefore suggest that there may be a discontinuity of the first derivative of $c$ in the Neveu-Schwatz sector at $r_{2}$. The analysis of $r_{4}$ shows similar but less marked properties. We could not get any information about other $r_{2 n}^{l}$. However if there is indeed a singularity for $r_{2}$ and $r_{4}$ it seems natural there is one at all $r_{2 n}$. Recall that in the polymer language, at $r=r_{2 n}$ it becomes possible for $2 n$ lines to propagate 
along the cylinder in time direction, and that this interferes with the characteristic feature of the ground state in the UV limit, the domination by non-contractible loops in space direction (with weight 2).

To investigate further the possibility of a singularity at $r_{2}^{l}$ we studied in detail the structure of the transfer matrix in the Neveu-Schwarz sector for complex $\beta^{l}$. For finite lattice size $R^{l}$, the largest eigenvalue does not cross for $\beta^{l}$ real. One can however locate square root branch points in the complex plane, a pair of which moves (in the blown-up variable $r^{l}$ ) towards $r_{2}^{l}$. The situation here is somewhat simpler than for ordinary critical phenomena, since in the variable $r^{l}$ the infinite number of branch points that converge to the critical inverse temperature $\beta_{c}^{l}$ are now at finite distance from each other. Hence we have to consider the limit point of a single pair of branch points, which is expected to exhibit a singularity of the form $x^{k}|x|$ (see [55] for more detailed discussion). This agrees with the above expectation of $k=0$. However a singularity of the largest eigenvalue is not enough here; we need to make sure that it occurs in its scaling part. We unfortunately did not get definitive numerical evidence for this.

\subsection{Miscellaneous}

We have two additional observations. At the UV and IR fixed points, we have $h=$ $g \mathrm{~m}^{2} / 4$ in the NS sector. Thus we can define the running coupling constant $g\left(r^{l}\right)$ by measuring the gap between the ground state of the NS sector and the ground state of the sector where two non-contractible loops propagate $(m=2)$. The result for available sizes is a monotonic curve with a derivative maximum at a value close to $g=1$, close also to $r_{4}^{l}$ (these coincidences do not seem to be exact).

We have also investigated whether the model passes "close" to an intermediate fixed point by studying the spectrum of excited states and looking for the tower structure characteristic of a conformal field theory. No such structure has been observed.

\section{Conclusions}

We have explained why $n$ negative was expected to exhibit even more complicated properties, and we shall not discuss it further. For $n \geq 0$, the key features of the large- $t$ expansion are reproduced by the various numerical studies. In particular, we emphasize that despite the complex nature of the interaction, the ground state energy of the sineGordon model (except for $n=-2$ ) remains real all along the flow. We have also checked 
that the levels of the sine-Gordon model that contribute to the minimal partition coincide with predictions from the massless TBA for minimal models. We have presented a detailed study of the case $n=0$ because supersymmetry allowed us to do so. This case is certainly a little marginal, since for instance the associated $c=0$ "minimal model" has no degrees of freedom and a partition function equal to one, and so is clearly "infinitely far" from the non-minimal one whose partition function grows exponentially. This case presents strong evidence for genuine singularities in the flow whose physical interpretation is rather clear from polymer point of view. The natural questions to ask now are

1) are there really singularities in the flow for $n=0$ ?

2 ) if there are indeed singularities for $n=0$, is it a marginal case or do all $0 \leq n$ values exhibit the same behavior?

Certainly for $0<n<2$ the situation is somewhat different than for $n=0$ since the free energies of minimal and sine-Gordon models are then the same (level crossings occur in some sectors, but only in finite numbers).

3 ) is there an exact scattering theory for massless flow within sine-Gordon and between successive minimal models, at least for some values of $0 \leq n$ ?

The study of these questions and partial answers are presented in the next paper.

\section{Acknowledgments}

We would like to thank K. Intriligator, G. Moore, C. Vafa and A.B. Zamolodchikov for useful conversations. H.S. was supported by the Packard Foundation and DOE grant DE-AC-76ERO3075, while P.F. was supported by DOE grant DEAC02-89ER-40509.

\section{Appendix A. Polymer results}

We summarize here the polymer results obtained in this paper for the reader with this specific interest only. Consider the total number of configurations for a set of $L$ polymer lines of total number of edges $\mathcal{N}$ crossing the cylinder of radius $R^{l}$ (which we denote simply by $R$ here). One has

$$
\Omega_{L}^{(R)} \propto\left(\mu_{L}^{(R)}\right)^{\mathcal{N}}, \mathcal{N}>>1
$$

The connectivity constant $\mu_{L}^{(R)}$ is such that for every $L, \mu_{L}^{(R)} \rightarrow \mu$ as $R \rightarrow \infty$ where $\mu$ is the usual lattice-dependent "bulk" connectivity constant [56]. Using finite-size scaling one has $\mu_{L}^{(R)}-\mu \propto R^{-1 / \nu}$, where $\nu=3 / 4$. Define the numbers (where $\beta_{c}=1 / \mu$ )

$$
\lim _{R \rightarrow \infty}\left(1 / \mu_{L}^{(R)}-\beta_{c}\right)^{\nu} R^{l}=r_{L}^{l}
$$


These numbers are universal up to a lattice-dependent overall scale (that is $r_{L} / r_{L^{\prime}}$ is universal). We have found that the particular numbers $r_{2+4 n}$ are in the same ratio as the (simple) $n^{\text {th }}$ poles of the function $Q$ given by $(6.5) ; Q$ is the derivative of a solution of the Painlevé III equation (6.4), with boundary condition (6.6). The locations of these poles do not have simple expressions. The first few numerical values are

$\begin{array}{lllll}1^{\text {st }} & 2^{\text {nd }} & 3^{\text {rd }} & 4^{\text {th }} & 5^{\text {th }} \\ 2.95708396 & 8.61 & 14.1 & 19.5 & 24.8\end{array}$

We have also found that the distance $r_{2+4 n}-r_{-2+4 n}$ behaves as $C r_{4 n}$ for $n$ large, where $C$ is a constant close to $C \simeq 5$.

That $Q$ should have a singularity can also be established on physical grounds using the polymer model. Indeed recall that in 21] we showed that

$$
Q(r)=\int_{0}^{\infty} e^{-(r)^{4 / 3} x} f(x) d x
$$

where the number of configurations of a single non-contractible loop of length $\mathcal{N}$ on the cylinder reads in the scaling limit

$$
\omega_{R}=T R^{-7 / 3} \mu^{\mathcal{N}} s f\left(\frac{\mathcal{N} s}{R^{4 / 3}}\right) .
$$

and we have set

$$
r=\left(\frac{\beta_{c}-\beta}{s \beta_{c}}\right)^{3 / 4} R
$$

while $s$ is some lattice-dependent rescaling factor. The dominant behavior of the function $f$ follows from the loss of entropy due to confinement that is $f(y) \simeq y^{\alpha} \exp (-c y)$ where $\alpha, c$ are constants to be determined. Plugging this result back into the Laplace transform (A.3) we see immediately that $c=\left(r_{2}\right)^{4 / 3}$ and $\alpha=0$. That the connectivity constant for a non-contractible loop and for two propagating legs $(L=2)$ should be the same appears reasonable, and it is proven by the quantum-group arguments in the text.

Some of these results could likely be extended to the other number of legs, in particular odd ones. However the connection with the PIII equation, which has been our main analytical tool here, is possible only for a number of legs equal to two modulo four, which is characteristic of the Ramond sector in the $N=2$ point of view. 


\section{References}

[1] A.B. Zamolodchikov, JETP Lett. 43 (1986) 730.

[2] A. Ludwig, J. Cardy, Nucl. Phys. B285 (1987) 687.

[3] Al.B. Zamolodchikov, Nucl. Phys. B358 (1991) 497, 524.

[4] V. Dotsenko, V.A. Fateev, Nucl. Phys. B240 (1984) 312.

[5] T. Eguchi, S.K. Yang, Phys. Lett. 224B (1989) 373.

[6] T. Hollowood, P. Mansfield, Phys. Lett. 226B (1989) 73.

[7] B. Nienhuis, J. Stat. Phys. 34 (1984) 153.

[8] G. Felder, Nucl. Phys. B317 (1989) 215.

[9] C. Gomez, G. Sierra, Phys. Lett. B240 (1990) 149.

[10] P. Bouwknegt, J. McCarthy, K. Pilch, Nucl. Phys. B352 (1991) 139.

[11] T. Eguchi, S.K. Yang, in Proceedings of the eighth symposium on theoretical physics, Kyohak Yunkusa, Ed. H. S. Song (1989).

[12] N.Yu. Reshetikhin, F.A. Smirnov, Comm. Math. Phys. 131 (1990) 157.

[13] A. LeClair, Phys. Lett. B230 (1989) 103.

[14] D. Bernard, A. LeClair, Comm. Math. Phys. 142 (1991) 99.

[15] G. Felder, A. LeClair, Int. J. Mod. Phys. A7 (1992) 239.

[16] V. Pasquier, Comm. Math. Phys. 118 (1988) 355.

[17] V. Pasquier, H. Saleur, Nucl. Phys. B330 (1990) 523.

[18] A.B. Zamolodchikov, Mod. Phys. Lett. A6 (1991) 1807.

[19] F.A. Smirnov, Phys. Lett. B275 (1992) 109.

[20] M.T. Grisaru, A. Lerda, S. Penati and D. Zanon, Phys. Lett. B234 (1990) 88; Nucl. Phys. B342 (1990) 564.

[21] P. Fendley, H. Saleur, Nucl. Phys. B388 (1992) 609.

[22] S. Cecotti, C.Vafa, Nucl. Phys. B367 (1991) 359.

[23] S. Cecotti, P. Fendley, K. Intriligator, C. Vafa, Nucl. Phys. B386 (1992) 405.

[24] A.A. Belavin, A. Polyakov, A.B. Zamolodchikov, Nucl. Phys. B241 (1984) 333.

[25] A.B. Zamolodchikov and Al.B. Zamolodchikov, Ann. Phys. 120 (1980) 253

[26] D. Boyanovsky, R. Holman, Nucl. Phys. B332 (1990) 641.

[27] J.V. Jose, L.P. Kadanoff, S. Kirkpatrick, D.R. Nelson, Phys. Rev. B16 (1977) 1217.

[28] J. Cardy, in "Fields, Strings and Critical Phenomena", Les Houches Proceedings (1988), ed. by E. Brezin and J. Zinn-Justin, North Holland.

[29] B. Nienhuis, Physica 163A (1990) 152; B. Nienhuis, H. Blöte, J. Phys. A22 (1989) 1415.

[30] P. Martin, "Potts Models and Related Problems in Statistical Mechanics", World Scientific, 1991.

[31] R.J. Baxter, J. Phys. A19 (1986) 2821.

[32] B. Nienhuis, Phys. Rev. Lett. 49 (1982) 1062. 
[33] N.Yu. Reshetikhin, J.Phys. A24 (1991) 2387.

[34] H. Blöte, J. Cardy, M.P. Nightingale, Phys. Rev. Lett. 56 (1986) 742.

[35] F. Alcaraz, M.N. Barber, T.M. Batchelor, Phys. Rev. Lett. 58 (1987) 771.

[36] P. DiFrancesco, H. Saleur, J.B. Zuber, J. Stat. Phys. 49 (1987) 57.

[37] A. Cappelli, C. Itzykson, J.B. Zuber, Nucl. Phys. B280 (1987) 445.

[38] F. Ravanini, Phys. Lett. 274B (1992) 345.

[39] C.N. Yang, C.P. Yang, J. Math. Phys. 10 (1969) 1115.

[40] Al. B. Zamolodchikov, Nucl. Phys. B342 (1990) 695.

[41] I. Affleck, Phys. Rev. Lett. 56 (1986) 746.

[42] W. Press, B. Flannery, S. Teukolsky and W. Vetterling, "Numerical Recipes" (Cambridge, 1989)

[43] I. Kostov, M. Staudacher, Nucl. Phys. B384 (1992) 459.

[44] C. Itzykson, H. Saleur, J. Stat. Phys. 48 (1987) 449.

[45] T. Klassen, E. Melzer, Nucl. Phys. B350 (1990) 635.

[46] H. Blöte, B. Nienhuis, Physica A160 (1989) 121.

[47] H. Saleur, Nucl. Phys. B382 (1992) 486.

[48] G. Waterson, Phys. Lett. B171 (1986) 77

[49] R. Balian, G.Toulouse, Ann. Phys. 83 (1974) 28.

[50] H. Saleur, Ph.D. Thesis, Paris 6 University.

[51] E. Witten, Nucl. Phys. B202 (1982) 253.

[52] A.B. Zamolodchikov, Sov. J. Nucl. Phys. 44 (1986) 529;

D. Kastor, E. Martinec, S. Shenker, Nucl. Phys. B316 (1989) 590;

E. Martinec, Phys. Lett. 217B (1989) 431;

C. Vafa, N.P. Warner, Phys. Lett. 218B (1989) 51.

[53] B.M. McCoy, C.A. Tracy, T.T Wu, J. Math. Phys. 18 (1977) 1058.

[54] M. Cvetic, D. Kutasov, Phys. Lett. B240 (1990) 1502.

[55] D.W. Wood, J.Phys. A 20 (1987) 3471; D.W. Wood et al., J.Phys. A 20 (1987) 3495.

[56] A.J. Guttman, J.Phys. A17 (1984) 455, and references therein. 


\section{Figure Captions}

Figure 1: Data for the running central charge $c(m R)$ for $n=\sqrt{2}$ obtained from the lattice model and analytic continuation of massive TBA

Figure 2: Data for the running central charge at $n=-1$ obtained from the lattice model. Observe the appearance of additional extrema in the IR as $R^{l}$ increases.

Figure 3: The locus of zeroes for the Ising model partition function. Perturbation by a purely imaginary mass moves along the circles.

Figure 4a: A set of $L$ polymer lines propagating on the cylinder

Figure 4b: Growth of an infinite arm at $r_{2}$

Figure 5: Discontinuity of the running subtracted central charge in the Ramond sector $(n=0)$ at $r_{2}$

Figure 6: Running central charge $c(m R)$ for $n=0$ obtained from the lattice

Figure 7: Second derivative of $c(r=m R)$ with respect to $r$ for $n=0$. 


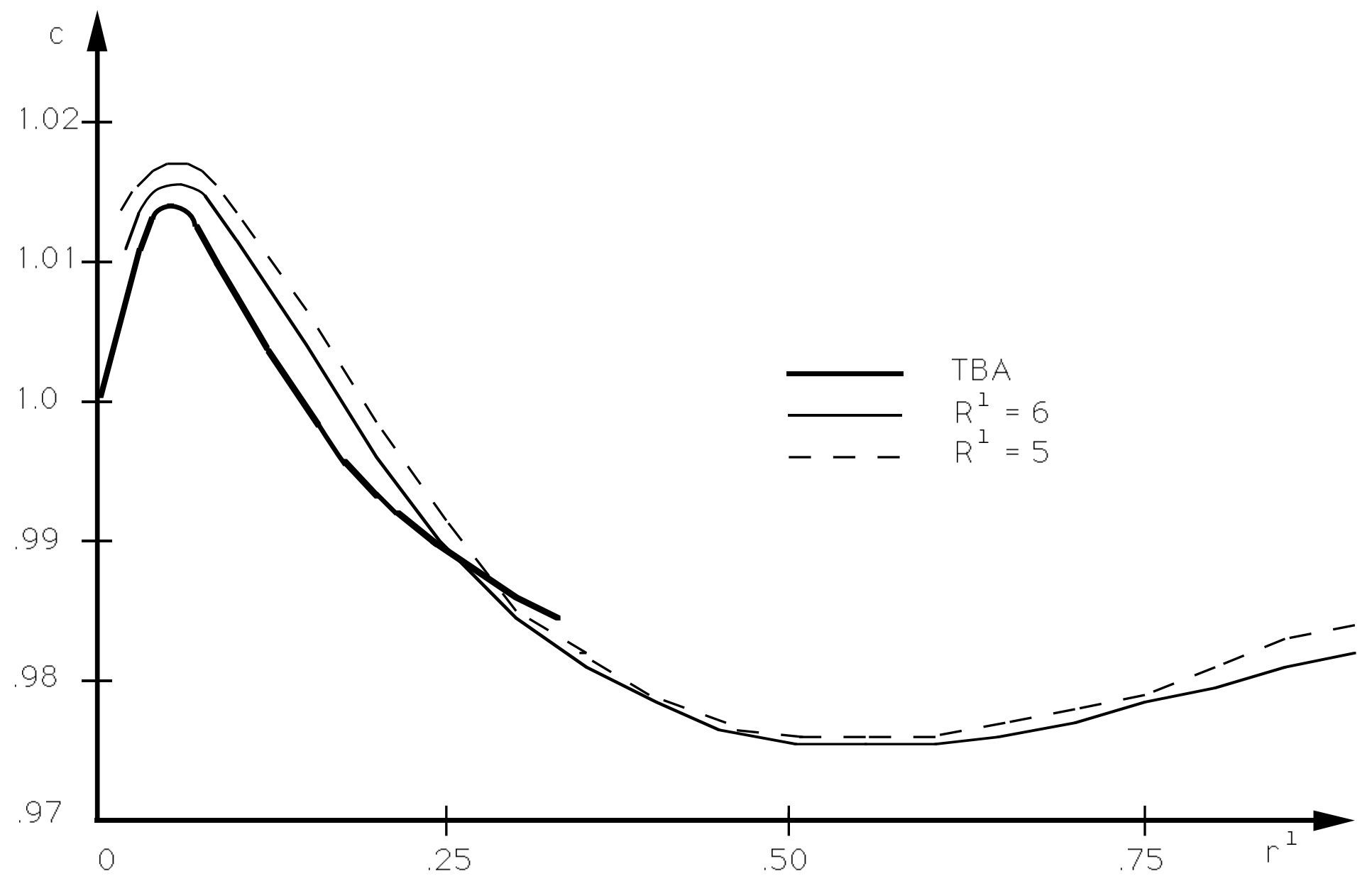

Fig. 1. Comparison of TBA and lattice data for $n=\sqrt{2}$ 


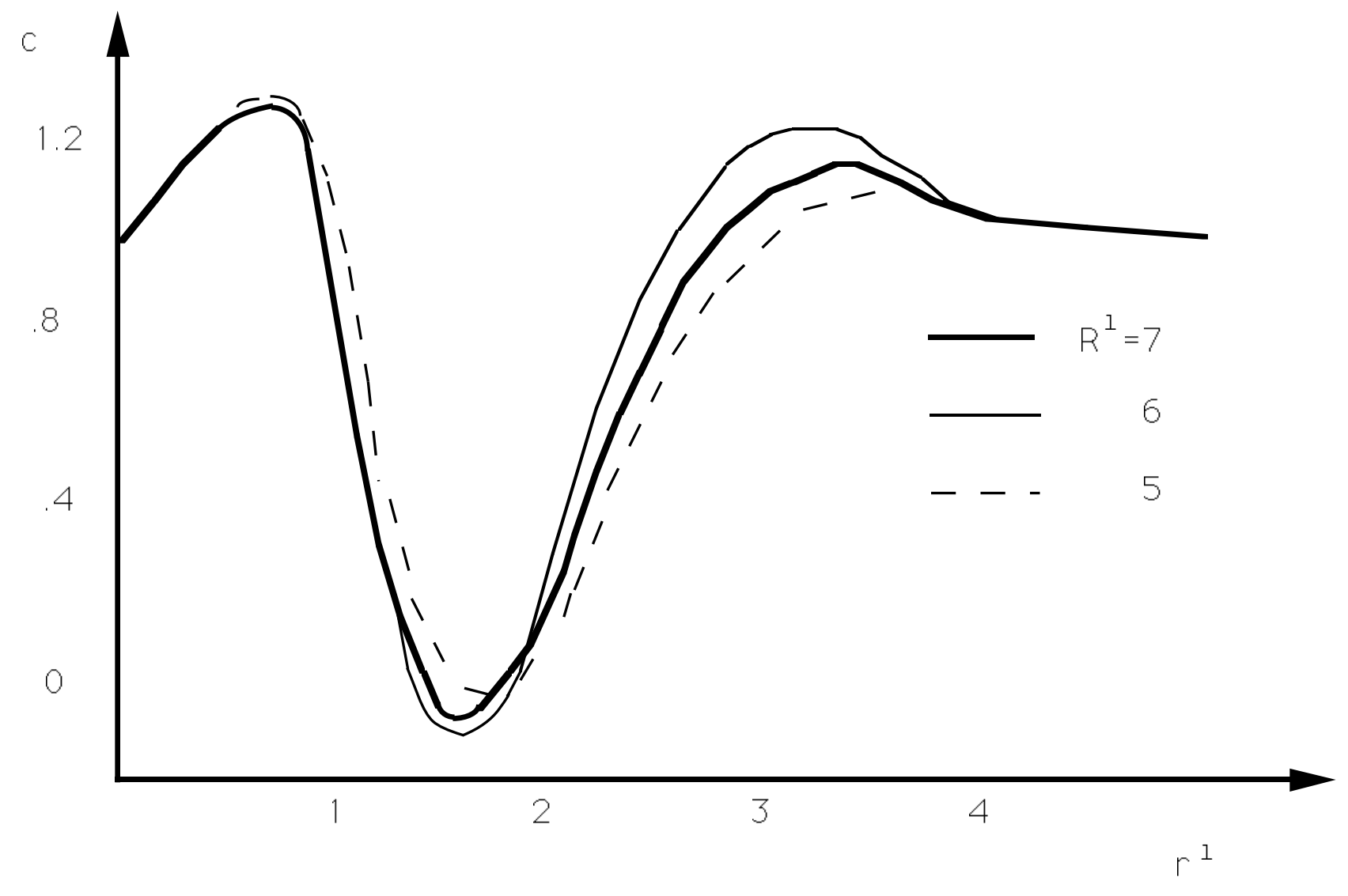

Fig. 2. $c(r)$ for $n=-1$ 


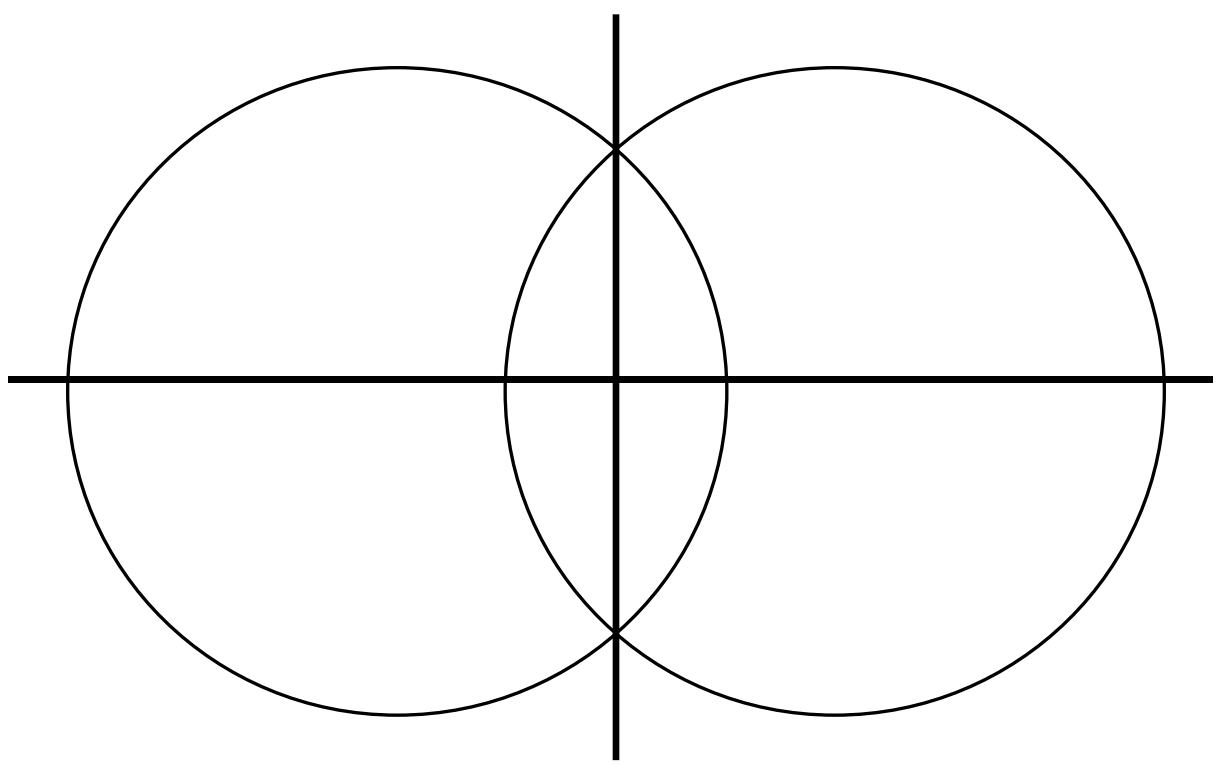

FIg. 3. Locus of I sing model zeros 


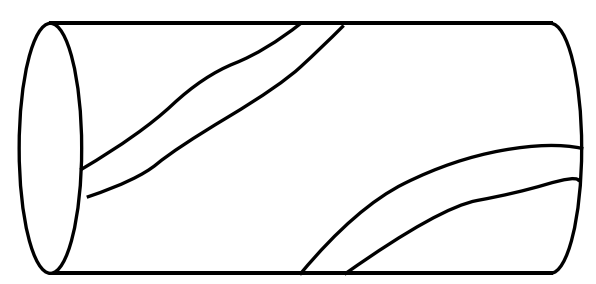

Fig. 4a. Two polymers propagating together

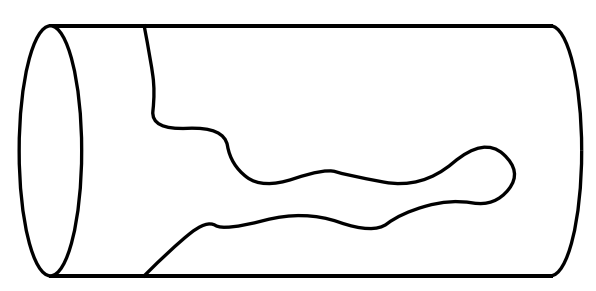

Fig. 4b. Growth of an infinite arm 


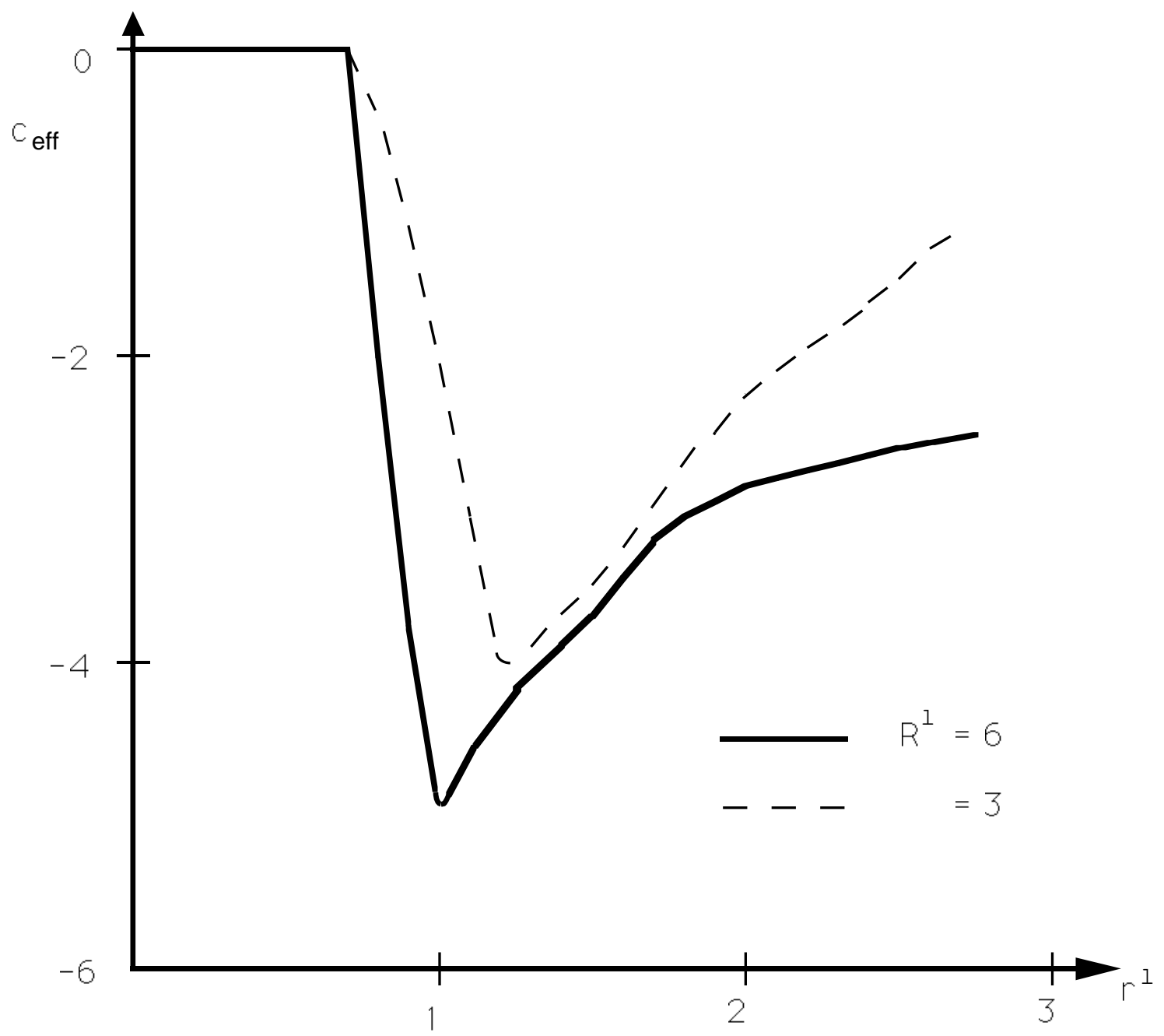

Fig, 5, $c_{\text {eff }}$ in Ramond sector for $n=0$ 

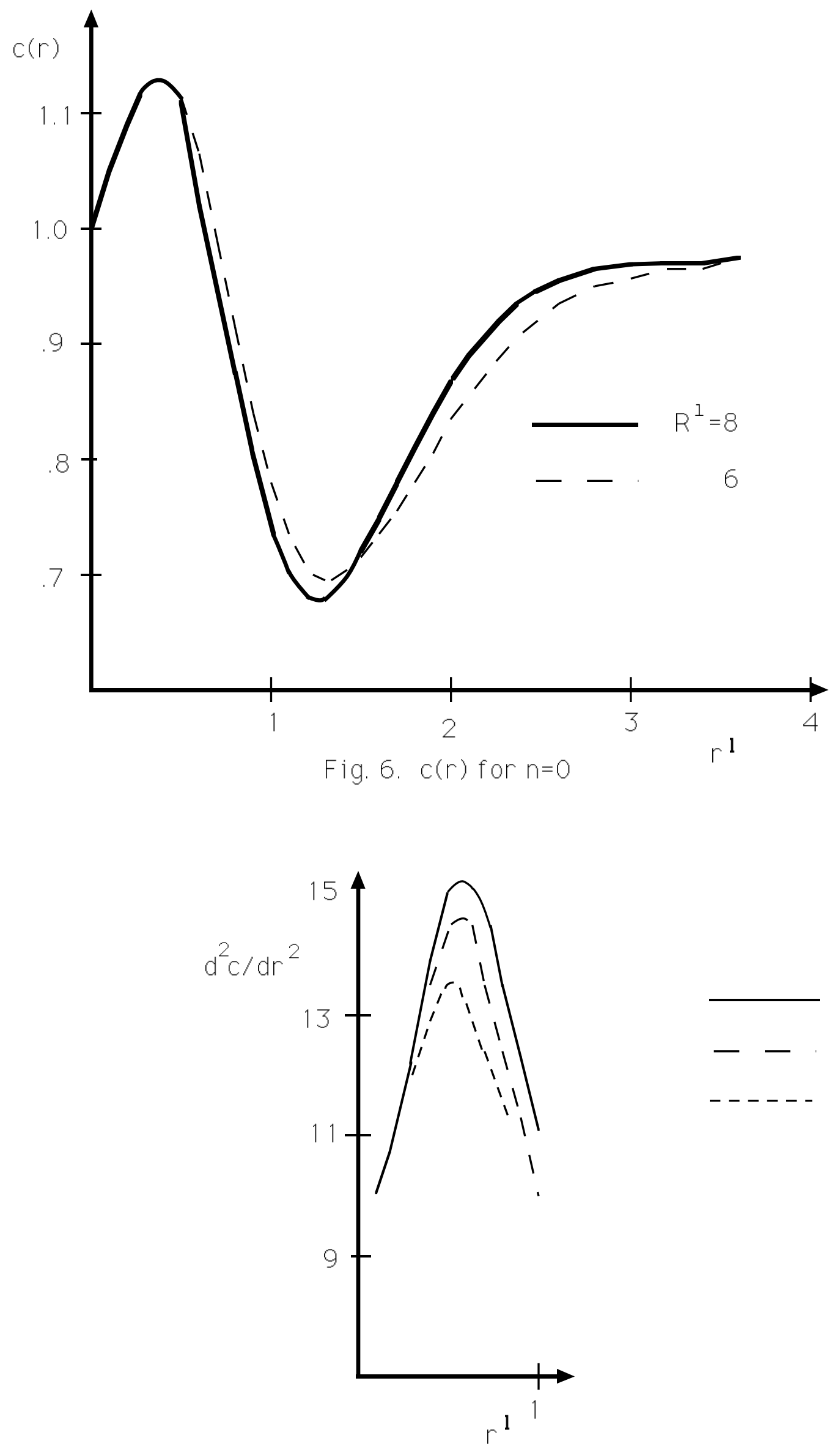

$R^{1}=7$

6

5

Fig. 7. Derivative for $n=0$ 